\title{
An Isomonodromy Cluster of Two Regular Singularities
}

\author{
A. V. Kitaev * \\ Steklov Mathematical Institute, Fontanka 27, St.Petersburg, 191023, Russia \\ and \\ School of Mathematics and Statistics, University of Sydney, \\ Sydney, NSW 2006, Australia
}

August 28, 2018

\begin{abstract}
We consider a linear $2 \times 2$ matrix ODE with two coalescing regular singularities. This coalescence is restricted with an isomonodromy condition with respect to the distance between the merging singularities in a way consistent with the ODE. In particular, a zero-distance limit for the ODE exists. The monodromy group of the limiting ODE is calculated in terms of the original one. This coalescing process generates a limit for the corresponding nonlinear systems of isomonodromy deformations. In our main example the latter limit reads as $P_{6} \rightarrow P_{5}$, where $P_{n}$ is the $n$-th Painlevé equation. We also discuss some general problems which arise while studying the above-mentioned limits for the Painlevé equations.
\end{abstract}

2000 Mathematics Subject Classification: 34M55, 33E17, 33E30

Short title: Isomonodromy Cluster

Key words: Isomonodromy deformations, asymptotics, Schlesinger transformations, Painlevé equations

\footnotetext{
*E-mail: kitaev@pdmi.ras.ru
} 


\section{Prelude}

This work appeared first as Sfb 288 Preprint No. 149 (Teschnishe Universität, Berlin) in December 1994. It was distributed to all leading mathematical institutions and many researchers. It was submitted to Comm. Math. Phys. (November 1994), but was not accepted as "not interesting for the readership" of that Journal. The copy of this preprint in various formats can be downloaded from CERN Document Server ${ }^{1}$. During the past (more than) 11 years I presented this work in a number of talks on various conferences, colloquium talks, and many private discussions. During this period appeared also a number of works by different authors that discuss questions closely related with this work. A review of those works actually requires considerable space and time. So, I was forced to avoid this issue. The only changes I made in the bibliography is the inclusion of the brief announcement [18, of the works [19, 20] and also an appropriate renumbering of the references.

In spite of the development that have been achieved during the past decade, the main results obtained in my preprint were not reproduced. Moreover, the intensive development of the Random Matrix Theory and related topics in the theory of Orthogonal Polynomials reveals that various double scaling limits of the Painlevé equations, one of those we study in this work, play a significant role in various questions of these theories and applications.

So, I believe that the publication of this work will be helpful as presenting an important property of the nonlinear special function - the sixth Painlevé equation $\left(P_{6}\right)$ and for future studies of isomonodromy deformations.

I decided not to make any changes into the mathematical content of this work, just correction of English and misprints in formulae. Since it past so much time from the date the work was written, it is important to mention that the discretization procedure of the transition limit that studied in this work was motivated by the author's joint work with Alexander Its and Athanasis Fokas on matrix models and orthogonal polynomials 19, 20.

I am very grateful to the Guest Editors of this volume, Nalini Joshi and Frank Nijhoff, for giving me an opportunity to publish this work. I am also indebt to Fedor Andreev who typed the original version of this manuscript ${ }^{1}$ in $\mathcal{A M}_{\mathcal{M}}-\mathrm{T}_{\mathrm{E}} \mathrm{X}$ and Arthur Vartanian for helping me in correction of English.

\section{Introduction}

By the isomonodromy cluster of two regular singularities we mean two points in the complex plane $\mathbb{C}$ with respective positions $\lambda_{i}(t)(i=0,1)$, considered as given functions of the parameter $t$ defined in a neighborhood $\mathcal{O}\left(t_{0}\right) \subset \mathbb{C}$ (or $\mathbb{R}$ ) of some fixed point $t_{0} \in \mathbb{C}$ (or $\mathbb{R}$ ), satisfying the following conditions: (1) $\lambda_{0}\left(t_{0}\right)=\lambda_{1}\left(t_{0}\right) ;(2) \lambda_{0}(t) \neq \lambda_{1}(t)$ for $t \in \mathcal{O}\left(t_{0}\right) \backslash t_{0}$; and (3) they are the simple poles of the linear ODE

$$
\frac{d}{d \lambda} \Psi=\left(\frac{A_{0}(t)}{\lambda-\lambda_{0}(t)}+\frac{A_{1}(t)}{\lambda-\lambda_{1}(t)}+A(\lambda, t)\right) \Psi
$$

where $A_{0}(t), A_{1}(t), A(\lambda, t) \in \operatorname{Mat}(n, \mathbb{C})$ are analytic functions of $t$, and $A(\lambda, t)$ is a rational function of $\lambda$. It is also assumed that there exists a fundamental solution of Eq. (2.1) with the manifold of monodromy data ${ }^{2}$ independent of $t$ (hard isomonodromy condition).

\footnotetext{
${ }^{1}$ http://preprints.cern.ch/cgi-bin/ setlink?base $=$ preprint\&categ $=. \& i d=$ SCAN-9501196

${ }^{2}$ The manifold of monodromy data is defined in Section 3
} 
Thus Eq. (2.1) is equipped with a system of nonlinear ODEs (with respect to $t$ ) governing isomonodromy deformations of its coefficients.

For the simplest nontrivial backgrounds (the functions $A(\lambda, t)$ ), the systems of isomonodromy deformations can be reduced to classical Painlevé equations; in particular, in this work we consider the fifth Painlevé equation $P_{5}\left(n=2, \lambda-0=0, \lambda_{1}=t\right.$, $A(\lambda, t)=$ const $\left.\cdot \sigma_{3} \neq 0\right)$ and the sixth one $P_{6}\left(n=2, \lambda_{0}=0, \lambda_{1}=t, A(\lambda, t)=A(t) /(\lambda-1)\right.$, $A_{0}(t)+A_{1}(t)+A(t)=$ const $\left.\cdot \sigma_{3} \neq 0\right)$.

The main idea in studying the cluster system (2.1), equipped with the isomonodromy condition, is to substitute into Eq. (2.1), instead of the cluster entries, some singularity of a regular or irregular type. Carrying out this procedure in accordance with the isomonodromy condition, we obtain, instead of (2.1), a new equation, whose fundamental solution solution is denoted by $\Psi_{\text {new }}$, and a novel system of isomonodromy deformations with respect to a parameter which is proportional to $t-t_{0}$. As a result we obtain a formal limit (or formal asymptotics) as $t \rightarrow t_{0}$ of the initial Eq. (2.1) to a new one, and of the initial system of isomonodromy deformations to a novel one. To translate this formal limit into the nonformal result, i.e., to understand the solutions of the new system of isomonodromy deformations as defining the master term of asymptotic expansions as $t \rightarrow t_{0}$ for the solutions of the initial system of isomonodromy deformations, one has to solve the following nontrivial problem: to calculate the manifold of monodromy data for $\Psi_{\text {new }}$ in terms of the monodromy manifold for $\Psi$. This is the problem addressed here by taking the background in Eq. (2.1) corresponding to $P_{6}$. The latter result is easy to generalize to an arbitrary number of regular singularities.

It was M. Jimbo [1] who considered isomonodromy cluster of two regular singularities for the linear ODEs associated with $P_{5}$ and $P_{6}$. In accordance with the aforementioned, Jimbo's work can be interpreted as follows: Jimbo considered Eq. (2.1) with $P_{5}$ and $P_{6}$ backgrounds and substituted for the cluster terms a regular singularity. The resulting ODE for $\Psi_{\text {new }}$, by means of simple gauge and scaling transformations, can be reduced to matrix versions of the standard ODEs for the Gauss hypergeometric function in the case of $P_{6}$, and the Whittaker confluent hypergeometric function in the case of $P_{5}$. From this, one observes:

1. The coefficients of the standard ODEs, which are constant (with respect to $t$ ) parameters, define the manifolds of monodromy data for the Gauss hypergeometric and Whittaker functions and, thereby, define the manifold of monodromy data for $\Psi_{\text {new }}$;

2. The coefficients of the standard ODEs also define coefficients of the ODE for $\Psi_{\text {new }}$, the latter coefficients, which define solutions of the new system of isomonodromy deformations, asymptotically, as $t \rightarrow 0$ behave as linear combinations of power-like functions, $t^{\alpha}$.

Because the deformations are isomonodromic, it is easy to relate the monodromy manifolds of the initial function $\Psi$ with the monodromy manifolds of the standard equations. Thus, having done items 1 and 2 for each Painlevé equation, $P_{5}$ and $P_{6}$, Jimbo was able to parameterize the small- $t$ asymptotics for $P_{5}$ and $P_{6}$ by points of the corresponding manifolds of monodromy data; for $P_{6}$, i.e., when Eq. (2.1) has, along with the cluster $\{0, t\}$, two additional singularities at 1 and $\infty$, Jimbo considered the group of fractionallinear transformations of $\mathbb{C}$ which interchange the cluster with the singularities 1 and 
$\infty$. Having found the action of this group on the manifold of monodromy data, he obtained connection formulae for asymptotic expansions of general solutions of $P_{6}$ as $t \rightarrow 0$, $t \rightarrow 1$, and $t \rightarrow \infty$. Subsequently, McCoy and Tang [2, 3, 4] used Jimbo's monodromy parametrization for small- $t$ asymptotics of $P_{5}$ to connect them with the large- $t$ asymptotic expansions for the same equation.

Like Jimbo [1, we consider here the cluster on the $P_{6}$ background; however, unlike the hard isomonodromy condition used by Jimbo, we introduce a weak one, i.e., the deformations which preserve the generators of the monodromy group, forming an essential part of the manifold of monodromy data, rather than the whole manifold as for the hard isomonodromy deformations. As we see later, the condition that isomonodromy deformations are weak but not hard implies their discretization. At the same time the weak isomonodromy condition gives us an opportunity to substitute for the cluster not only the regular singularity (as in the Jimbo case) but also an irregular one. In this work we consider two different clusters on the $P_{6}$ background: the first one described by an irregular singularity and the other by a regular one. Even the "regular" case studied here differs from that in [1]; in particular, it is related with a formal limit $P_{6} \rightarrow P_{5}$, the "irregular" case leads to another formal limit $P_{6} \rightarrow P_{5}$. The main goal of this paper is to give a proper asymptotic interpretation of the formal limits in the manner explained above.

We will study the first limit passage of the following oriented graph,

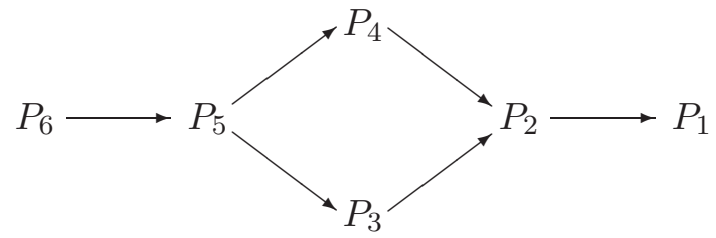

Figure 1: The Painlevé - Okamoto degeneration scheme

which represents the successive degeneration of the Painlevé equations $\left(P_{n}\right)$. This degeneration scheme was obtained by Painlevé [5], who showed that by substituting into the canonical Painlevé equations a parameter $\epsilon$ and transforming the subsequent equations via appropriate $\epsilon \rightarrow 0$ limits, simpler Painlevé equations can be obtained ${ }^{3}$. Okamoto [6] pointed out that these formal limits can be viewed as infinitesimal canonical transformations of the Hamiltonian systems associated with the Painlevé equations; thus he equipped the scheme of Figure 1 with the analogous scheme for Hamiltonians. Furthermore, Okamoto associated with this step-by-step degeneration process a step-by-step confluence scheme for certain scalar linear ODEs, whose isomonodromy deformations are governed by the Painlevé equations. These scalar equations are known to be intimately connected with matrix equations of the form (2.11). The degeneration scheme for the linear ODEs can be formulated in a manner analogous to that shown in Figure 1, in terms of a special symbol which represents the corresponding transformation of the Poincaré ranks of singularities of the linear ODEs.

The authors works [7, 8, 9] in conjunction with the present one can be understood as the isomonodromy regularization of the degeneration scheme of Figure 11 In addition to the scheme discussed above, our regularization suggests the following scheme of transfor-

\footnotetext{
${ }^{3}$ The scheme was extensively studied by Okamoto, who also presented it as the graph on Figure 1.
} 
mation for the manifolds of monodromy data of the associated linear matrix ODEs:

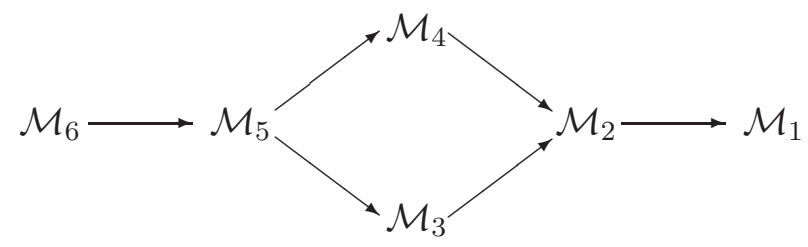

Figure 2

Let us now discuss the schemes in Figures 1 and 2 in more detail. Each oriented edge in the Figures means the existence of a corresponding limit passage; in fact, there could also exist some other limits; for example, the limit $P_{6} \rightarrow P_{5}(t \rightarrow 1)$, found by Painlevé [5], differs from both of ours (in which $t \rightarrow 0$ ). We call the formal limits equivalent if the diagram in Figure 3 is commutative, where the $x$-arrows denote the action of the transformation groups on the solutions of the Painlevé equations (for $n=6$, $m=5$, see [10, 11]) or the manifolds of monodromy data, the $y$-arrows denote the same formal limits as on Figures 1 and 2 respectively, and where the $z$-arrows "enumerate" the solutions by the points of the manifolds of monodromy data, i.e., they "point out" on solutions of the corresponding direct monodromy problems (see Section 3 ).

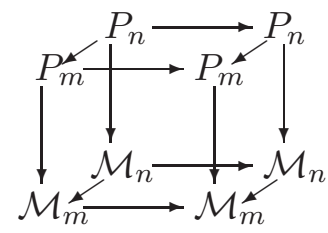

Figure 3

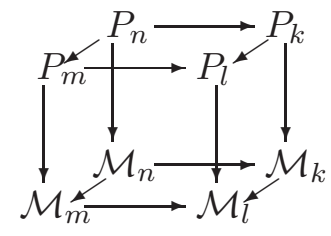

Figure 4

Note that if the transformation $P_{n} \rightarrow P_{n}$ acts not only between the initial Painlevé equations but also during the entire limiting process, i.e., for arbitrary $\epsilon \rightarrow 0^{4}$, then the entire $\mathcal{M}$-plane of Figure 3 is not needed, since this commutativity is valid automatically. In general, there are some transformations of the Painlevé equations which do not have this property, i.e., they cannot be extended for arbitrary $\varepsilon$, we present the diagram in extended form. Although, as explained above, the limits $P_{6} \rightarrow P_{5}$ that we study in this work have different asymptotic senses, they appear to be equivalent under the above definition; moreover, all limits $P_{6} \rightarrow P_{5}$ known as at the time of the presents are equivalent. Therefore, the following question arises naturally: are there an $n$ and $m$ such that nonequivalent formal limits $P_{n} \rightarrow P_{m}$ exist?

There are some Painlevé equations which are known to be equivalent; for example, $P_{5}^{\prime}\left(P_{5}\right.$ with $\delta_{5}=0$, see (3.56) $)$ is equivalent to $P_{3}$ [12] and $P_{34}$ is equivalent to $P_{2}$ [13]. This equivalence of the equations means that there is a mapping which is invertible on transcendental solutions and birationally dependent of them and their derivatives. Along with the limit $P_{4} \rightarrow P_{2}$ (see Figure 1), the formal limit $P_{4} \rightarrow P_{34}$ was found in [7. Are these limits "substantially" different?

To formulate the general notion of equivalence of the formal limits of different Painlevé equations, i.e., the equivalence of the limits $P_{n} \rightarrow P_{m}$ and $P_{k} \rightarrow P_{l}$, we consider Figure 4.

\footnotetext{
${ }^{4}$ More precisely, if we denote by $\varepsilon$ and $\varepsilon_{1}$ small parameters in two copies of $P_{n}$, then the transformation maps $\varepsilon \rightarrow \varepsilon_{1}$, such that the functions $\varepsilon\left(\varepsilon_{1}\right) \rightarrow 0$ and $\varepsilon_{1}(\varepsilon) \rightarrow 0$, when $\varepsilon_{1}$ or, respectively, $\varepsilon \rightarrow 0$.
} 
In this figure the upper $x$-arrows denote the birational mappings (which are assumed to exist). The mappings $\mathcal{M}_{n} \rightarrow \mathcal{M}_{k}$ and $\mathcal{M}_{m} \rightarrow \mathcal{M}_{l}$ are uniquely defined by demanding commutativity for the $x z$-walls of the cube. The $y$-arrows denote the equivalence classes of the limits defined earlier via Figure 3. The $z$-arrows have the same meaning as that in Figure 3. The limits $P_{n} \rightarrow P_{m}$ and $P_{k} \rightarrow P_{l}$ are said to be equivalent if the diagram in Figure 4 is commutative. Are there any nonequivalent limits for pairwise equivalent equations? In particular, are the limits $P_{4} \rightarrow P_{2}$ and $P_{4} \rightarrow P_{34}$ equivalent? Although according to [14 these limits show different actions on local expansions for solutions of $P_{4}$, they can appear to be equivalent under the above definition.

The cluster point of view makes clear that there must exist limits other than those shown in Figure 1 e.g., along with the chain of successive limits $P_{4} \rightarrow P_{2} \rightarrow P_{1}$, there exist direct ones [7, 8, which look, at first glance, to be different from the limit obtained via the successive procedure. Thus we can expect the existence of a more complicated limit structure (than that shown on Figure 10, which can be presented as the following oriented spatial graph:

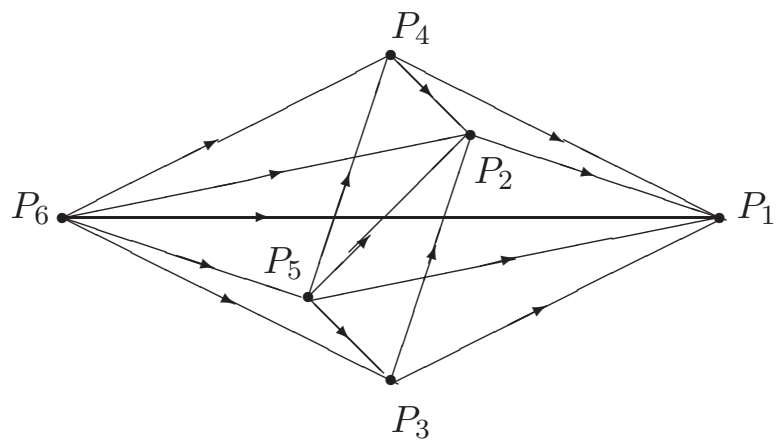

Figure 5: A spatial degeneration scheme for the Painlevé equations

Furthermore, it seems that the vertices $P_{2}$ and $P_{3}$ could have a more complicated microlocal structure $P_{2} \sim P_{2} \rightleftarrows P_{34}$ and $P_{3} \sim P_{3} \rightleftarrows P_{5}^{\prime} \rightleftarrows P_{3}^{\prime}$ where $P_{3}^{\prime}$ is a special case of $P_{3}$ defined by the following conditions: $\gamma \delta=0$ and $|\gamma|+|\delta| \neq 0$, where $\gamma$ and $\delta$ are the coefficients of the canonical form of $P_{3}$ [13. If this is the case, then some of the edges which are incident to $P_{3}$ must be paired off in order to be incident to $P_{3}$ and $P_{3}^{\prime}$. The answers to our "equivalence" questions (see above) could lead to a further complication of the diagram in Figure 5. The answer to the first question governs the number of its edges, and the answer to the second question could result in additional vertices and edges (say, $P_{n} \rightarrow P_{34}$ and $P_{n} \rightarrow P_{5}^{\prime}$ ). In Figure 5 we observe that for most of the pairs of vertices there are several routes connecting them. The natural question therefore is: are there any nonequivalent routes for some pair of vertices? The notion of equivalent routes, $P_{n} \rightarrow P_{k} \rightarrow P_{l}$ and $P_{n} \rightarrow P_{m} \rightarrow P_{l}$, can also be formulated by means of the commutativity of the diagram in Figure 4. For this purpose we must identify the roles of the $x$ - and $y$-arrows, understanding them as denoting the equivalence classes of the limits. For routes with three or four edges this definition must, naturally, be generalized.

As mentioned, the original setting of the formal limits for ODEs of the Painlevé type includes the small parameter $\varepsilon$. The appearance of $\varepsilon$ endows the problem with an infinite-dimensional status, as the constants of integration can be considered as arbitrary functions of $\varepsilon$. Furthermore, it is possible that the latter functions do not have a limit as $\varepsilon \rightarrow 0$, although the corresponding limiting equations exist and therefore possess no 
explicit $\varepsilon$ dependence. Thus, we see that for a proper understanding of such limits on the level of the solutions a regularization procedure may be required. The regularization procedure, which we adopt in this work, means that we will be making such choices of the functions mentioned above so that they would satisfy the weak isomonodromy condition. This requirement leads us (see [7, 8, 9] and Sections [5] and 6) to the discretization of $\varepsilon$. It is clear that one could propose different regularization procedures for the formal limits: different procedures depend predominantly on possible applications and/or the employed mathematical machinery. Of course, some regularizations could require no $\varepsilon$-discretization. In particular we mean the approach proposed by Joshi and Kruskal [14. It is necessary to mention at once that not all the details are set out explicitly in [14; therefore, 14] yields a variety of different possibilities: here, we consider one such possibility. Consider the Laurent expansion about a neighborhood of a pole for a solution of some Painlevé equation. It has the following parameters: (coefficients of the Painlevé equation; (2) one more constant of integration along with the position of the pole; and (3) dependent and independent variables, i.e., the Painlevé function itself and its argument. It is possible to define transformation of all the above parameters (at least, again, as formal Laurent-type $\varepsilon$-expansions near $\varepsilon=0$ ) which map the initial Laurent expansion for the solution of the Painlevé equation into a new one. The latter, in turn, can be considered as a pole expansion of the solution of some other (limiting) Painlevé equation. All the parameters of the new pole expansion are defined by the coefficients of the leading terms of the formal $\varepsilon$-expansions. These leading terms are precisely the formulae given by Painlevé in his original work [5]. So in [14] no $\varepsilon$-discretization and any associated linear structures and thereby manifolds of monodromy data are involved.

We can provide Joshi's and Kruskal's work [14] with, perhaps, a somewhat unexpected interpretation, which yields an opportunity to relate their method with isomonodromy deformations. This interpretation lead us, again, to a discretization of $\varepsilon$; but in this case, in a way different from that proposed in [7], and below in Sections [5] and [6] Recall that all the Painlevé equations are known to possess transcendental solutions having an infinite number of poles, $\left\{t_{n}\right\}$, on the real axis accumulating at infinity. The above $\varepsilon$ transformation can be interpreted as a procedure for driving the poles of solutions of the Painlevé equation to $\infty$. We fix a particular solution, which has an infinite number of poles accumulating at infinity, and treat the "pole-drive" as a pole-to-pole jumping. This leads to a discretization of $\varepsilon\left(\varepsilon \rightarrow \varepsilon_{n}\right)$, because we are aloud now to do only discrete shifts of the poles $t_{n} \rightarrow t_{n+1}$. Simultaneously iterating the pole expansion via Bäcklund transformations by the number of times consistent with the $\varepsilon$-transformation, we arrive at the pole expansion of some particular solution of the limiting Painlevé equation. The consistency means that we take the number of Bäcklund transformations such that the coefficients of the corresponding Painlevé equation increase by a rate prescribed by the formulae for $\varepsilon$-transformation (with $\varepsilon \rightarrow \varepsilon_{n}$ ). In other words, on the level of the pole expansions, we can interpret the limiting passage between Painlevé equations as some special asymptotics of poles of (fixed) solutions. These asymptotics, in turn, are given in terms of a pole of some particular solution of the limiting Painlevé equation. Note that our interpretation of the limiting passage is consistent with isomonodromy deformations. We know that in the standard situation (without Bäcklund iterations) pole-asymptotics of solutions can be parameterized by the monodromy data (see [15]). Is that possible to get an analogous parametrization of the poles in the new situation described above ${ }^{5}$ ?

\footnotetext{
${ }^{5}$ The latter parametrization, in fact, means that the monodromy data of the initial Painlevé equation
} 
It is important to make a distinction with our previous works [7, 8, 9, which were concerned with the WKB-method and where the clusters of turning points were considered, and this work, where no WKB-method is involved. Hence, now, we are considering a different asymptotic process. In general, for Painlevé equations all limiting procedures are related with various processes of merging turning points and singularities in the associated linear ODEs describing corresponding isomonodromy deformations. For example, the limit $P_{4} \rightarrow P_{2}$ could be organized by considering the following two possibilities for the asymptotic behavior of the $2 \times 2$ matrix linear ODE associated with $P_{4}$ : (1) merging of four turning points; and (2) merging of the irregular and regular singular points, i.e., in our terminology, we can consider either an isomonodromy cluster of four turning points or an isomonodromy cluster of irregular and regular singularities. So it seems that, really, nonequivalent limits could exist, and the graph on Figure 5 could actually be incomplete.

The discretization of $\varepsilon$ is not too important a feature of the technique we are developing. Actually, in the isomonodromy systems with more than one continuous variable, the so-called higher Painlevé equations or Garnier systems, we can observe the analogous asymptotic processes and apply the same technique without any $\varepsilon$-discretization [16]. We mention also an interesting WKB-theory for special solutions of the Painlevé equations and its interrelation with the corresponding WKB objects for the associated scalar linear ODEs [17.

The technique considered in [7, 8, 9, and here has an interesting scope of applications. One meets the clusters of turning points while studying via the isomonodromy approach double-scaling limits of partition functions for matrix models of $2 D$ quantum gravity [19, 20. Clusters of stationary phase (saddle) points, which are similar to the clusters of turning points, appear in the description of caustics in $1+1$ systems integrable via the Inverse Scattering Transform [16]. The clusters of regular singularities play an important role in the study of a zero-curvature limit for holonomic quantum field theory of bosons in the Poincare disc 21, 22] ${ }^{6}$. The limit relates this theory with the original Euclidean SatoMiwa-Jimbo theory [23, 24, 25, 26, 27, 28. The correspondence of two-point correlators of these theories in our notation reads as $P_{6} \rightarrow P_{3}$ or $P_{6} \rightarrow P_{5}^{\prime}$. Considering Figure 5 we see that if we add to the limit $P_{6} \rightarrow P_{5}$ the next one $P_{5} \rightarrow P_{3}$, then we obtain the desired limit $P_{6} \rightarrow P_{3}$. The limit $P_{5} \rightarrow P_{3}$ in our approach is also related with the isomonodromy cluster of two regular singularities but on the "irregular background" $\left(A(\lambda, t)=\right.$ const $\left.\cdot \sigma_{3} \neq 0\right)$. Of course, there is a direct limit $P_{6} \rightarrow P_{3}$, and the question of equivalence discussed above should be studied. I hope to return to this question in a special publication.

This paper is organized as follows: Section 3 contains no new material. It is based mainly on the works [1, 29, 30. In this Section we set the notation related with the description of isomonodromy deformations for $P_{6}$ and $P_{5}$. Most of the formulae presented here are extensively used throughout Sections 4,6. Some of our definitions are slightly different from those of the cited works; in particular, I found it convenient to define two different manifolds of monodromy data for $P_{5}\left(\mathcal{M}_{5}\right.$ and $\left.\tilde{\mathcal{M}}_{5}\right)$ : they are related with the schemes of the calculations in Sections 5 and 6. namely, constructions of semi-infinite sequences of Schlesinger transformations, which are important issues in our approach.

In Section 4, we follow the simple procedure explained at the beginning of the Introduction to derive two different and novel formal limits $P_{6} \rightarrow P_{5}$. The corresponding

are related with the monodromy data of the limiting one.

${ }^{6}$ Cited works are written from a different perspective 
formulae for the canonical Painlevé functions are rather cumbersome, whilst those being rewritten for the corresponding $\tau$-functions become much simpler.

In Section 5 we derive a discrete regularized version of the first limit. We calculate the manifold of the monodromy data for the limiting $P_{5}$ transcendent in terms of the corresponding manifold for the initial $P_{6}$ transcendent. The result is stated in Theorem 5.1, and the following part of the section presents details of the derivation.

Theorem 6.1 of Section [6] states results for a discrete regularization of the second limit analogous to those in Section 5 however, a derivation of these results is more complicated than those in Section 4, because, in Section [6 we meet an additional problem with the normalization of a priori unknown function $\Psi_{5}$. It causes a number of additional technical detail one of them is considered in Appendix. As a by-product for these efforts, we get: (1) a clear picture of how the monodromy matrices of the merging regular singularities produce the Stokes multipliers of the resulting $\Psi_{5}$-function which has an irregular singularity describing the merging process; and (2) the latter derivation is easy to generalize for a background with an arbitrary number of regular singularities.

In the case of $P_{6}$, when Equation (2.1) has four regular singularities, the cluster and exterior cluster domains can be mapped one into another via a fractional-linear transformation. The latter means a transformation which maps the cluster of Section 5 into the one of Section [6] Thus we prove the equivalence of the limits for the Painlevé transcendents and the corresponding $\tau$-functions.

In the Appendix we study the problem of the simultaneous reduction of a pair of $\mathrm{SL}(2, \mathbb{C})$ matrices to the lower and upper triangular forms. The solution is stated in a theorem which refers to a number of special cases studied in corresponding propositions. These results have an important consequence on the solvability of the asymptotic problem studied in Section (6). A generalization of the problem of the simultaneous transformation of $n \geq 3 \mathrm{SL}(2, \mathbb{C})$ matrices to triangular forms is important for the investigation of the isomonodromy clusters with $n$ regular singularities.

\section{$3 \quad P_{6}$ and $P_{5}$ as Isomonodromy Deformations}

In this section we recall some facts from the isomonodromy theory of $P_{6}$ and $P_{5}$ following, mainly, the works [1, 29, 30]. To make a distinction between analogous objects related to $P_{n}(n=5,6)$ for different $n$, we supply all of them by the subscript $n$. The convenience of this agreement becomes evident in the following sections.

Consider the $2 \times 2$ matrix linear ODE with four regular singularities at $\lambda=0,1, t, \infty$ :

$$
\frac{d}{d \lambda} \Psi_{6}=\left(\frac{A_{06}}{\lambda}+\frac{A_{16}}{\lambda-1}+\frac{A_{t 6}}{\lambda-t_{6}}\right) \Psi_{6}
$$

It is assumed that

$$
A_{06}+A_{16}+A_{t 6}=-\frac{\Theta_{\infty 6}}{2} \sigma_{3}, \quad \sigma_{3}=\left(\begin{array}{cc}
1 & 0 \\
0 & -1
\end{array}\right), \quad \Theta_{\infty 6} \in \mathbb{C} \backslash \mathbb{Z},
$$

and there exist $R_{\nu 6} \in \mathrm{SL}(2, \mathbb{C})$ such that

$$
R_{\nu 6}^{-1} A_{\nu 6} R_{\nu 6}=\frac{\Theta_{\nu 6}}{2} \sigma_{3}, \quad \Theta_{\nu 6} \in \mathbb{C} \backslash \mathbb{Z} .
$$


In a neighborhood of the regular singularity $\lambda=\nu(\nu \neq \infty)$, the $\Psi_{6}$-function can be expanded as

$$
\Psi_{6} \underset{\lambda \rightarrow \nu}{=} \sum_{k=0}^{\infty} \Psi_{k \nu 6}(\lambda-\nu)^{k+\frac{\Theta_{\nu 6}}{2} \sigma_{3}} C_{\nu 6},
$$

where the matrices $\Psi_{k \nu 6}, C_{\nu 6}$ are independent of $\lambda$, because of the normalization conditions (3.2) and (3.3), we can assume that

$$
\Psi_{6}, R_{\nu 6}, \Psi_{0 \nu 6}, C_{\nu 6} \in \mathrm{SL}(2, \mathbb{C}), \quad \nu=0,1, t .
$$

Because of Equation (3.2), the expansion of the $\Psi_{6}$-function can be normalized at $\lambda=\infty$ :

$$
\Psi_{6} \underset{\lambda \rightarrow \infty}{=}\left(I+\sum_{k=1}^{\infty} \Psi_{k \infty 6} \lambda^{-k}\right) \lambda^{-\frac{\Theta_{\infty} 6}{2} \sigma_{3}} .
$$

The single-valued $\Psi_{6}$-function with the stated properties can be defined on the $\lambda$-plane "cut" along the negative imaginary semi-axis $[-i \infty, 0]$, the segment $\left[0, t_{6}\right]$, and the positive real semi-axis $[1,+\infty]$. Henceforth, we assume that $t_{6} \in(0,1)$. Following [1] we choose the paths in $\overline{\mathbb{C}} \backslash\left\{0, t_{6}, 1, \infty\right\}$ as is shown on Figure 6 . Continuing the $\Psi_{6}$-function along these

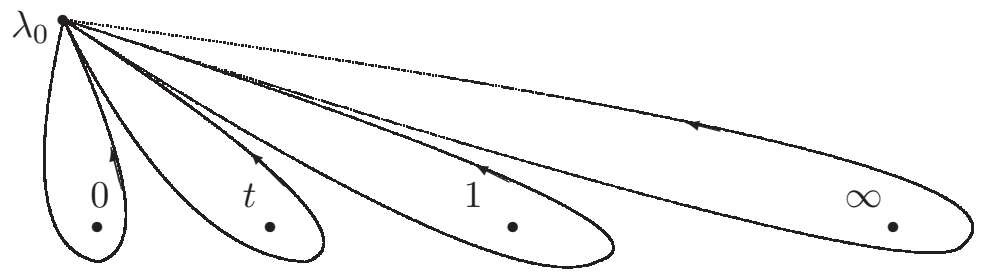

Figure 6

paths we find a multi-valued function with the monodromy matrices $M_{\nu 6}(\nu=0,1, t, \infty)$ which, in terms of the local expansions (3.4) and (3.6), can be written as follows:

$$
M_{\nu 6}=C_{\nu 6}^{-1} e^{\pi i \Theta_{\nu 6} \sigma_{3}} C_{\nu 6}, \quad \nu=0,1, t, \infty, \quad C_{\infty 6}=I .
$$

One proves the cyclic relation

$$
M_{\infty 6} M_{16} M_{t 6} M_{06}=I .
$$

The monodromy group $\left(\mathcal{M G}_{6}\right)$ is the subgroup of $\mathrm{SL}(2, \mathbb{C})$ generated by the matrices $M_{\nu 6}$. The set of monodromy data $\left(\mathcal{M}_{6}\right)$ is an ordered set of matrix elements of $M_{\nu 6}$ completed with four complex parameters $\Theta_{\nu 6}(\nu=0,1, t, \infty)$, satisfying the equation

$$
\operatorname{tr} M_{\nu 6}=2 \cos \left(\pi \Theta_{\nu 6}\right) .
$$

The numbers $\Theta_{\nu 6}$ are called the coefficients of formal monodromy.

Thus we see that for a fixed parameter $t_{6}$ and matrices $A_{\nu 6}$ the above procedure yields the unique set $\mathcal{M}_{6}$. One proves that a different set of $\left\{A_{\nu 6}\right\}_{\nu=0,1, t}$, with the same $t_{6}$, defines a different set $\mathcal{M}_{6}$; therefore, considering the Riemann problem of reconstruction of all differential equations corresponding to the given set $\mathcal{M}_{6}$, we have to vary not only $A_{\nu 6}$, but also $t_{6}$. Following the Schlesinger approach it is convenient to consider $t_{6}$ as an independent variable, then $A_{\nu 6}=A_{\nu 6}\left(t_{6}\right)$. To find these functions explicitly one has 
to notice that the hard isomonodromy condition, i.e., $\partial_{t_{6}} \mathcal{M}_{6}=0$, leads to the additional ODE with respect to $t_{6}$ for the function $\Psi_{6}$, which, under the assumptions on the formal monodromy (3.2) and (3.3), reads

$$
\frac{\partial}{\partial t_{6}} \Psi_{6}=-\frac{A_{t 6}}{\lambda-t_{6}} \Psi_{6}
$$

The compatibility condition of Equations (3.1) and (3.10) is

$$
\frac{d A_{\nu 6}}{d t_{6}}=\frac{\left[A_{t 6}, A_{\nu 6}\right]}{t_{6}-\nu}, \quad \nu=0,1
$$

where $[A, B] \equiv A B-B A$. In the derivation of (3.11) we took into account the normalization condition (3.2) together with $\partial_{t_{6}} \Theta_{\infty 6}=0$.

The system (3.11) is called the system of isomonodromy deformations or the Schlesinger system. It consists of eight scalar nonlinear ODEs of the first order for the matrix elements of $A_{\nu 6}(\nu=0,1)$. By using Equations (3.2) and (3.3), one finds the following five first integrals:

$$
\operatorname{tr} A_{\nu 6}=0, \quad \operatorname{det} A_{\nu 6}=-\frac{\Theta_{\nu 6}^{2}}{4}, \nu=0,1, \quad \operatorname{det}\left(\Theta_{\infty 6} \sigma_{3} / 2+A_{06}+A_{16}\right)=-\Theta_{t 6}^{2} / 4,
$$

reducing the number of independent scalar equations to three. This number coincides with with the complex dimension of the manifold of monodromy data, $\mathcal{M}_{6}\left(\Theta_{06}, \Theta_{16}, \Theta_{t 6}, \Theta_{\infty 6}\right)$. The points of this manifold are in one-to-one correspondence with the set $\mathcal{M}_{6}$ for the fixed parameters $\Theta_{\nu 6}$. More precisely: identifying an ordered set of the matrix elements of $\mathcal{M}_{6}$ as a point in $\mathbb{C}^{14}$, one defines $\mathcal{M}_{6}\left(\Theta_{06}, \Theta_{16}, \Theta_{t 6}, \Theta_{\infty 6}\right)$ as an algebraic variety in $\mathbb{C}^{14}$ by Equations (3.8), (3.9), and $\operatorname{det} M_{\nu 6}=1,(\nu=0,1, t, \infty)$.

Following [30], introduce the notation,

$$
A_{\nu 6}=\left(\begin{array}{cc}
z_{\nu 6}+\frac{\Theta_{\nu 6}}{2} & -u_{\nu 6} z_{\nu 6} \\
\frac{z_{\nu 6}+\Theta_{\nu 6}}{u_{\nu 6}} & -z_{\nu 6}-\frac{\Theta_{\nu 6}}{2}
\end{array}\right), \quad R_{\nu 6}=\left(\begin{array}{cc}
\frac{1}{\Theta_{\nu 6} s_{\nu 6}} & u_{\nu 6} z_{\nu 6} s_{\nu 6} \\
\frac{1}{\Theta_{\nu 6} u_{\nu 6} s_{\nu 6}} & \left(z_{\nu 6}+\Theta_{\nu 6}\right) s_{\nu 6}
\end{array}\right),
$$

where $\nu=0,1, t$, and $s_{\nu 6} \in \mathbb{C} \backslash\{0\}$. Equation (3.2) in the notation (3.12) reads:

$$
\begin{gathered}
z_{06}+z_{16}+z_{t 6}=-\frac{1}{2}\left(\Theta_{06}+\Theta_{16}+\Theta_{t 6}+\Theta_{\infty 6}\right), \\
u_{06} z_{06}+u_{16} z_{16}+u_{t 6} z_{t 6}=0, \quad \frac{z_{06}+\Theta_{06}}{u_{06}}+\frac{z_{16}+\Theta_{16}}{u_{16}}+\frac{z_{t 6}+\Theta_{t 6}}{u_{t 6}}=0 .
\end{gathered}
$$

Thus, only three parameters amongst those which define the coefficients of Equation (3.1) are independent. In the hard isomonodromy case the parameters appear to be functions of $t_{6}: z_{\nu 6}=z_{\nu 6}\left(t_{6}\right)$ and $u_{\nu 6}=u_{\nu 6}\left(t_{6}\right)$ defined by System (3.11). As explained above, one can rewrite (3.11) as a system of three scalar first-order ODEs. A convenient form of this system is given in [30. Here we define two important functions closely related with System (3.11). The first one,

$$
y_{6}=\frac{t_{6} u_{06} z_{06}}{\left(t_{6}+1\right) u_{06} z_{06}+t_{6} u_{16} z_{16}+u_{t 6} z_{t 6}}=\frac{1}{1+\left(1-\frac{1}{t_{6}}\right) \frac{u_{16} z_{16}}{u_{06} z_{06}}}=\frac{t_{6}}{1+\left(1-t_{6}\right) \frac{u_{t 6} z_{t 6}}{u_{06} z_{06}}},
$$


is a solution of the sixth Painlevé equation,

$$
\begin{gathered}
{\frac{d^{2} y_{6}{ }^{2}}{d t_{6}}}^{=}=\frac{1}{2}\left(\frac{1}{y_{6}}+\frac{1}{y_{6}-1}+\frac{1}{y_{6}-t_{6}}\right)\left(\frac{d y_{6}}{d t_{6}}\right)^{2}-\left(\frac{1}{t_{6}}+\frac{1}{t_{6}-1}+\frac{1}{y_{6}-t_{6}}\right) \frac{d y_{6}}{d t_{6}} \\
+\frac{y_{6}\left(y_{6}-1\right)\left(y_{6}-t_{6}\right)}{t_{6}^{2}\left(t_{6}-1\right)^{2}}\left(\alpha_{6}+\frac{\beta_{6} t_{6}}{y_{6}^{2}}+\frac{\gamma_{6}\left(t_{6}-1\right)}{\left(y_{6}-1\right)^{2}}+\frac{\delta_{6} t_{6}\left(t_{6}-1\right)}{\left(y_{6}-t_{6}\right)^{2}}\right) \\
\alpha_{6}=\frac{1}{2}\left(\Theta_{\infty 6}-1\right)^{2}, \quad \beta_{6}=-\frac{1}{2} \Theta_{06}^{2}, \quad \gamma_{6}=\frac{1}{2} \Theta_{16}^{2}, \quad \delta_{6}=\frac{1}{2}\left(1-\Theta_{t 6}^{2}\right) .
\end{gathered}
$$

The second function, which is important for applications, is the $\tau$-function [30]:

$$
\frac{d}{d t_{6}} \log \tau_{6}\left(t_{6}\right)=\operatorname{tr}\left(\frac{A_{06}}{t_{6}}+\frac{A_{16}}{t_{6}-1}\right) A_{t 6}
$$

The function

$$
\hat{\sigma}_{6}\left(t_{6}\right)=t_{6}\left(t_{6}-1\right) \frac{d}{d t_{6}} \log \tau_{6}\left(t_{6}\right)
$$

satisfies an ODE of the second order, quadratic with respect to $\hat{\sigma}_{6}^{\prime \prime}\left(t_{6}\right)$ [1, 6, 30] $]^{7}$. Conversely, starting from an arbitrary solution of Equation (3.16) and using the formulae given in [30] one can construct a solution of the Schlesinger System (3.11) satisfying Equation (3.15). Therefore, all solutions of $P_{6}$ (3.16) can be obtained from some solution of the Schlesinger System (3.11) via Equation (3.15).

Let us consider the weak isomonodromy deformations of Equation (13.1) as the deformations of $A_{\nu 6}(\nu, 0,1, t)$ preserving, up to the sign, the generators of $\mathcal{M G}_{6}$, i.e., isomonodromy deformations in the sense of the $\mathrm{PSL}_{2}(\mathbb{C})$ monodromy group. Using Equations (3.8) and (3.9) one proves that in the weak case the continuous Schlesinger deformations can be extended only by the group of discrete Schlesinger transformations acting on the formal monodromies as

$$
\Theta_{\nu 6} \rightarrow \Theta_{\nu 6}+n_{\nu}, \quad \nu=0,1, t, \infty, \quad n_{0}+n_{1}+n_{t}+n_{\infty}=0(\bmod 2),
$$

and the reflections

$$
\Theta_{\nu 6} \rightarrow-\Theta_{\nu 6}, \quad \nu=0,1, t
$$

Consider how these transformations act on the coefficients $A_{\nu 6}$ of Equation (3.1). First, notice that the reflections (3.20) are actually related with a certain ambiguity in the parametrization of the matrices $A_{\nu 6}$ rather than with any transformation of these matrices. The ambiguity in the parametrization is related with the ambiguity in writing the local expansion of the $\Psi_{6}$ function near the corresponding singular point: $\left(\Psi_{k \nu 6}, \Theta_{\nu 6}, C_{\nu 6}\right)$ $\mapsto\left(\Psi_{k \nu 6} \sigma_{1},-\Theta_{\nu 6}, \sigma_{1} C_{\nu 6}\right)$, where $\sigma_{1}$ is the Pauli matrix (with the matrix elements $\sigma_{1}^{i j}=0$ if $i=j$, else $\sigma_{1}^{i j}=1$ ). This nonuniqueness leads, simply, to the reparametrization of the matrices $A_{\nu 6}:\left(u_{\nu 6}, \Theta_{\nu 6}, z_{\nu 6}\right) \mapsto\left(\tilde{u}_{\nu 6}, \tilde{\Theta}_{\nu 6}, \tilde{z}_{\nu 6}\right)$, where $z_{\nu 6}+\frac{\Theta_{\nu 6}}{2}=\tilde{z}_{\nu 6}+\frac{\tilde{\Theta}_{\nu 6}}{2}, \tilde{\Theta}_{\nu 6}=\Theta_{\nu 6}$, and $u_{\nu 6} z_{\nu 6}=\tilde{u}_{\nu 6} \tilde{z}_{\nu 6}$, which doesn't affect the functions $y_{6}\left(t_{6}\right)$ and $\hat{\sigma}_{6}\left(t_{6}\right)$ (see Equations (3.15), (3.18), and (3.19) ).

Note that the reflection

$$
\tilde{\Theta}_{\infty 6}=-\Theta_{\infty 6}
$$

\footnotetext{
${ }^{7}$ In the cited papers, the authors use a definition of the $\sigma$-function shifted by a linear function of $t_{6}$. The latter function satisfies a differential equation symmetric with respect to the formal monodromies. We use a "hat" in our notation to make a difference between these functions.
} 
does not preserve the generators of $\mathcal{M G}_{6}$. This reflection is related with the change of the generators of $\mathcal{M G}_{6}$ to their inverse: $\widetilde{\mathcal{M G}}_{6}=\sigma_{1} \mathcal{M G}_{6} \sigma_{1}$. To see this one can define the following action of the reflection (3.22) on the $\Psi_{6}$ function, $\tilde{\Psi}_{6}=\sigma_{1} \Psi_{6} \sigma_{1}$; it yields:

$$
\tilde{M}_{\nu 6}=\sigma_{1} M_{\nu 6} \sigma_{1}, \quad \tilde{A}_{\nu 6}=\sigma_{1} A_{\nu 6} \sigma_{1} .
$$

It is an immediate consequence of Equations (3.23), (3.18), and (3.19) that $\tilde{\hat{\sigma}}_{6}=\hat{\sigma}_{6}$. In terms of the matrix elements the last equation in (3.23) reads:

$$
\begin{gathered}
\tilde{\Theta}_{\nu 6}= \pm \Theta_{\nu 6}, \quad \nu=0,1, t \\
\tilde{z}_{\nu 6}=-\left(z_{\nu 6}+\frac{\tilde{\Theta}_{\nu 6}+\Theta_{\nu 6}}{2}\right), \quad \tilde{u}_{\nu 6}=\frac{1}{u_{\nu 6}} \cdot \frac{z_{\nu 6}+\Theta_{\nu 6}}{z_{\nu 6}+\frac{\tilde{\Theta}_{\nu 6}+\Theta_{\nu 6}}{2}},
\end{gathered}
$$

where in Equation (3.24) for every value of $\nu$ we can make arbitrary choices of the signs. One can use Equations (3.25), 3.15), and Equations (C.49), (C.52), and (C.55) of [30] to find $\tilde{y}_{6}$ as a rational function of $y_{6}, y_{6}^{\prime}$, and $t_{6}$.

Consider the group of the discrete Schlesinger Transformations (3.20). Evidently, it has four generators and it is isomorphic to the group of translations along special basis in $\mathbb{C}^{4}$. We denote by $\mathcal{L}_{\nu \nu^{\prime}}^{ \pm \pm}$, where $\nu \neq \nu^{\prime}$, the elementary Schlesinger Transformations [30]:

$\mathcal{L}_{\nu \nu^{\prime}}^{ \pm \pm}: \quad \tilde{\Theta}_{\varkappa 6}=\Theta_{\varkappa 6} \pm 1, \quad$ for $\quad \varkappa=\nu, \nu^{\prime} ; \quad \tilde{\Theta}_{\varkappa 6}=\Theta_{\varkappa 6} \quad$ for $\quad \varkappa \in\{0,1, t, \infty\} \backslash\left\{\nu, \nu^{\prime}\right\}$.

In $\mathcal{L}_{\nu \nu^{\prime}}^{ \pm \pm}$we choose the signs over $\kappa=\nu, \nu^{\prime}$ in the same way as in the formula for $\tilde{\Theta}_{\varkappa 6}$. Since $\tilde{M}_{\varkappa 6}= \pm M_{\varkappa 6}$ (the sign minus occurs only for $\varkappa=\nu, \nu^{\prime}$ ), the action of $\mathcal{L}_{\nu \nu^{\prime}}^{ \pm \pm}$on the $\Psi_{6}$-function can be defined as the left multiplication

$$
\tilde{\Psi}_{6}=\mathcal{L}_{\nu \nu^{\prime}}^{ \pm \pm} \Psi_{6}
$$

where

$$
\begin{gathered}
L_{\nu \nu^{\prime}}^{ \pm \pm}=\frac{\sqrt{\lambda-\nu^{\prime}}}{\sqrt{\lambda-\nu}} J_{\nu \nu^{\prime}}^{ \pm \pm}+\frac{\sqrt{\lambda-\nu}}{\sqrt{\lambda-\nu^{\prime}}} J_{\nu^{\prime} \nu}^{ \pm \pm}, \quad \nu, \nu^{\prime}=0,1, t \\
L_{\nu \infty}^{ \pm \pm}=\sqrt{\lambda-\nu} \sigma_{\infty}^{ \pm}+\frac{1}{\sqrt{\lambda-\nu}} J_{\nu \infty}^{ \pm \pm}, \quad \nu \neq \infty, \quad \sigma_{\varkappa}^{+}=\left(\begin{array}{cc}
0 & 0 \\
0 & 1
\end{array}\right), \quad \sigma_{\varkappa}^{-}=\left(\begin{array}{ll}
1 & 0 \\
0 & 0
\end{array}\right) .
\end{gathered}
$$

Here, the branches of the roots are fixed as $\sqrt{\lambda-\nu} / \sqrt{\lambda} \rightarrow 1$ and $\sqrt{\lambda-\nu} / \sqrt{\lambda-\nu^{\prime}} \rightarrow 1$ as $\lambda \rightarrow \infty$ and $\lambda$ belongs to the complex plane cut as explained above (see the paragraph right after Equation (3.6)). The choice of the signs over all equal subscripts is the same (upper/lower). The matrices $J_{\nu \nu^{\prime}}^{ \pm \pm}$for $\nu, \nu^{\prime} \neq \infty$ are uniquely defined by the equations:

$$
J_{\nu \nu^{\prime}}^{ \pm \pm}+J_{\nu^{\prime} \nu}^{ \pm \pm}=I, \quad J_{\nu \nu^{\prime}}^{ \pm \pm} R_{\nu 6} \sigma_{\nu}^{\mp}=\left(\begin{array}{l}
0 \\
0
\end{array}\right)
$$

The result is as follows

$$
J_{\nu^{\prime} \nu}^{ \pm \pm}=\frac{1}{\Delta_{\nu^{\prime} \nu}^{ \pm}}\left(\begin{array}{cc}
b_{\nu}^{ \pm} & 0 \\
0 & -a_{\nu}^{ \pm}
\end{array}\right)\left(\begin{array}{cc}
a_{\nu^{\prime}}^{ \pm} & b_{\nu^{\prime}}^{ \pm} \\
a_{\nu^{\prime}}^{ \pm} & b_{\nu^{\prime}}^{ \pm}
\end{array}\right), \quad \Delta_{\nu^{\prime} \nu}^{ \pm}=a_{\nu^{\prime}}^{ \pm} b_{\nu}^{ \pm}-a_{\nu}^{ \pm} b_{\nu^{\prime}}^{ \pm},
$$

where $a_{\nu}^{ \pm}$and $b_{\nu}^{ \pm}$are different notations for the matrix elements of $R_{\nu 6}$ (see Equation (3.3)) which are convenient here, namely,

$$
R_{\nu 6}=\left(\begin{array}{cc}
b_{\nu}^{+} & b_{\nu}^{-} \\
-a_{\nu}^{+} & -a_{\nu}^{-}
\end{array}\right)
$$


The matrix $J_{\nu \nu^{\prime}}^{ \pm \pm}$can be found by the same Equations (3.27) and (3.28) by making the permutation of the subscripts $\left(\nu^{\prime} \leftrightarrow \nu\right)$. Note also the following useful properties of $J_{\nu \nu^{\prime}}^{ \pm \pm}$:

$$
\left(J_{\nu \nu^{\prime}}^{ \pm \pm}\right)^{2}=J_{\nu \nu^{\prime}}^{ \pm \pm}, \quad J_{\nu \nu^{\prime}}^{ \pm \pm} J_{\nu^{\prime} \nu}^{ \pm \pm}=0
$$

and analogous equations with $\nu \leftrightarrow \nu^{\prime}$.

We see that Transformation (3.26) exists iff $\Delta_{\nu \nu^{\prime}}^{ \pm} \neq 0$. Using Equations (C.51), (C.52), and (C.55) of [30] one finds that the condition $\Delta_{\nu \nu^{\prime}}^{ \pm}=0$ is equivalent to the existence of a one-parameter solution $y_{6}$ of $P_{6}$, which solves an ODE of the first order,

$$
\frac{d y_{6}}{d t_{6}}=R\left(y_{6}, t_{6}\right)
$$

where $R$ is a rational function of its arguments with the coefficients defined by $\Theta_{\nu 6}(\nu=$ $0,1, t, \infty)$. Thus, for general continuous Schlesinger deformations (3.11) which correspond to transcendental (nonclassical) solutions of $P_{6}$, the condition

$$
\Delta_{\nu \nu^{\prime}}^{ \pm} \neq 0
$$

is valid. Furthermore, we consider iterations of Transformations (3.26). If in some step we find $\Delta_{\nu \nu^{\prime}}^{ \pm}=0$, then it means we start from a solution of $P_{6}$ which is the iteration of a special (classical) solution of (3.16) , i.e., it can be presented in the form $\tilde{R}\left(y_{6}, y_{6}^{\prime}, t_{6}\right)$, where $\tilde{R}$ is a rational function of its arguments with the coefficients defined by $\Theta_{\nu 6}$ and where $y_{6}$ is a solution of an equation of the type (3.30). Thus we can iterate general (transcendental) solutions of $P_{6}$ (3.16) without any restrictions. The condition (3.31) is assumed throughout this paper. It would be interesting to perform the complete investigation of our problem including the special (classical) solutions of Equation (3.16). This investigation is in progress now [31, 32.

Let us also find the action of $\mathcal{L}_{\nu \nu^{\prime}}^{ \pm \pm}$on the matrices $A_{\nu 6}$ : substituting transformation (3.26) into Equation (3.16) written for $\tilde{A}_{\nu 6}$ and $\tilde{\Psi}_{6}$, one obtains:

$$
\begin{gathered}
J_{\nu^{\prime} \nu}^{ \pm \pm}\left(A_{\nu^{\prime} 6}-\frac{1}{2}\right)=\tilde{A}_{\nu^{\prime} 6} J_{\nu^{\prime} \nu}^{ \pm \pm}, \quad J_{\nu \nu^{\prime}}^{ \pm \pm}\left(A_{\nu 6}-\frac{1}{2}\right)=\tilde{A}_{\nu 6} J_{\nu \nu^{\prime}}^{ \pm \pm} \\
\tilde{A}_{\mu 6}=\left(J_{\nu^{\prime} \nu}^{ \pm \pm}+\frac{\nu-\mu}{\nu^{\prime}-\mu} J_{\nu \nu^{\prime}}^{ \pm \pm}\right) A_{\mu 6}\left(J_{\nu^{\prime} \nu}^{ \pm \pm}+\frac{\nu^{\prime}-\mu}{\nu-\mu} J_{\nu \nu^{\prime}}^{ \pm \pm}\right), \quad \mu \neq \nu, \nu^{\prime} .
\end{gathered}
$$

Using these equations and noting that the set $\left\{\nu, \nu^{\prime}, \mu\right\}$ is the permutation of $\{0,1, t\}$ we find

$$
\tilde{A}_{\nu 6}+\tilde{A}_{\nu^{\prime} 6}+\tilde{A}_{\mu 6}=-\frac{\Theta_{\infty 6}}{2} \sigma_{3}
$$

Multiplying Equation (3.34) by $J_{\nu \nu^{\prime}}^{ \pm \pm}$on the right and using Equations (3.29), (3.32), and (3.33), we find

$$
\begin{aligned}
\tilde{A}_{\nu^{\prime} 6}= & -\frac{\Theta_{\infty 6}}{2} \sigma_{3} J_{\nu \nu^{\prime}}^{ \pm \pm}+J_{\nu^{\prime} \nu}^{ \pm \pm}\left(A_{\nu^{\prime} 6}-\frac{1}{2}\right)-J_{\nu \nu^{\prime}}^{ \pm \pm}\left(A_{\nu 6}-\frac{1}{2}\right)- \\
& \left(\frac{\nu^{\prime}-\mu}{\nu-\mu} J_{\nu^{\prime} \nu}^{ \pm \pm}+J_{\nu \nu^{\prime}}^{ \pm \pm}\right) A_{\mu 6} J_{\nu \nu^{\prime}}^{ \pm \pm}
\end{aligned}
$$

where $\tilde{A}_{\nu 6}$ is given by the same Equation (3.35) but with the permutation $\nu^{\prime} \leftrightarrow \nu ; \tilde{A}_{\mu 6}$ can then be found from Equation (3.34). 
Consider, now, Transformation (3.26) for $\nu^{\prime}=\infty$ : we find that

$$
J_{\nu \infty}^{ \pm+}=\frac{1}{a_{\nu}^{ \pm}}\left(\begin{array}{cc}
1 & 0 \\
0 & -\Psi_{1 \infty 6}^{21}
\end{array}\right)\left(\begin{array}{cc}
a_{\nu}^{ \pm} & b_{\nu}^{ \pm} \\
a_{\nu}^{ \pm} & b_{\nu}^{ \pm}
\end{array}\right), \quad J_{\nu \infty}^{ \pm-}=\frac{1}{b_{\nu}^{ \pm}}\left(\begin{array}{cc}
-\Psi_{1 \infty 6}^{12} & 0 \\
0 & 1
\end{array}\right)\left(\begin{array}{cc}
a_{\nu}^{ \pm} & b_{\nu}^{ \pm} \\
a_{\nu}^{ \pm} & b_{\nu}^{ \pm}
\end{array}\right),
$$

where $a_{\nu}^{ \pm}$and $b_{\nu}^{ \pm}$are defined by Equations (3.28) and (3.12), $\Psi_{1 \infty 6}^{i j}$ are the matrix elements of the first coefficient of the expansion (3.6). These matrix elements can be calculated via the matrix elements of $A_{\nu 6}$, since (recall $\Theta_{\infty 6} \notin \mathbb{Z}$ ):

$$
-\Psi_{1 \infty 6}+\frac{\Theta_{\infty 6}}{2}\left[\sigma_{3}, \Psi_{1 \infty 6}\right]=A_{16}+t_{6} A_{t 6} .
$$

Thus, Transformation (3.26) with $\nu^{\prime}=\infty$ exists iff

$$
\mathcal{L}_{\nu \infty}^{ \pm+}: \quad a_{\nu}^{ \pm} \neq 0, \quad \mathcal{L}_{\nu \infty}^{ \pm-}: \quad b_{\nu}^{ \pm} \neq 0 .
$$

The violation of conditions (3.37) can be discussed in the same manner as the violation of Condition (3.31) (see the paragraph between Equations (3.29) and (3.32)). The only difference is that Equation (3.30) now takes the form

$$
\left(\frac{d y_{6}}{d t_{6}}\right)^{2}=R\left(y_{6}, t_{6}\right)
$$

Hereafter we assume that Conditions (3.37) are valid (as well as the previously assumed (3.31)).

Consider now the action of $\mathcal{L}_{\nu \infty}^{ \pm \pm}$on $A_{\mu 6}$, namely:

$$
\tilde{A}_{m u 6}=\left(\sigma_{\infty}^{ \pm}+\frac{1}{\mu-\nu} J_{\nu \infty}^{ \pm \pm}\right) A_{\mu 6}\left(\sigma_{\infty}^{ \pm}+\frac{1}{\mu-\nu} J_{\nu \infty}^{ \pm \pm}\right)^{-1}, \quad \mu \neq \nu
$$

The action of $\mathcal{L}_{\nu \infty}^{ \pm \pm}$on $A_{\nu 6}$ can be obtained by substituting Equation (3.38) for $\tilde{A}_{m u 6}$ and (with $\mu \leftrightarrow \mu^{\prime}$ ) for $\tilde{A}_{m u^{\prime} 6}$, where $\mu^{\prime}$ is defined from the condition that $\left\{\nu, \mu, \mu^{\prime}\right\}$ is a permutation of $0,1, t$, into the equation

$$
\tilde{A}_{\nu 6}+\tilde{A}_{\mu 6}+\tilde{A}_{\mu^{\prime} 6}=-\frac{\tilde{\Theta}_{\infty 6}^{ \pm}}{2} \sigma_{3}=-\frac{\Theta_{\infty 6} \pm 1}{2} \sigma_{3}, \quad \mu \neq \mu^{\prime} \neq \nu \neq \mu .
$$

To summarize, let us fix $t_{6}^{0} \in \mathbb{C} \backslash\{0,1\}$ and $A_{\nu 6}^{0}, \Theta_{\nu 6}^{0}$ as demanded by the last conditions in (3.2) and (3.3). The general Schlesinger (isomonodromy) deformations (GSD) for Equation (3.1) are the matrices $A_{\nu 6}$ (or their matrix elements) which depend on the continuous variable $t_{6}$ and the discrete variables $\Theta_{\nu 6}: \Theta_{\nu 6}-\Theta_{\nu 6}^{0} \in \mathbb{Z}, \sum_{\nu}\left(\Theta_{\nu 6}-\Theta_{\nu 6}^{0}\right)=$ $0(\bmod 2)$. The continuous deformations of $A_{\nu 6}$ are governed by (3.11) and the discrete deformations by Equations (3.33) - (3.35) and (3.38), 3.39). The initial condition is stated as $A_{\nu 6}\left(t_{6}, \Theta_{06}^{0}, \Theta_{16}^{0}, \Theta_{t 6}^{0}, \Theta_{\infty 6}^{0}\right)=A_{\nu 6}^{0}$. The continuous and discrete deformations are commuting so that GSD are correctly defined. Any GSD can be uniquely characterized by a point on $\mathcal{M}_{6}\left(\Theta_{06}^{0}, \Theta_{16}^{0}, \Theta_{t 6}^{0}, \Theta_{\infty 6}^{0}\right)$. The direct monodromy problem is: construct $\mathcal{M}_{6}\left(\Theta_{06}^{0}, \Theta_{16}^{0}, \Theta_{t 6}^{0}, \Theta_{\infty 6}^{0}\right)$ for the given GSD. The inverse monodromy problem is: construct the GSD for given $\mathcal{M}_{6}\left(\Theta_{06}^{0}, \Theta_{16}^{0}, \Theta_{t 6}^{0}, \Theta_{\infty 6}^{0}\right)$ [33.

Consider the $2 \times 2$ matrix linear ODE related with $P_{5}$ :

$$
\frac{d \Psi_{5}}{d \lambda}=\left(\frac{t_{5}}{2} \sigma_{3}+\frac{A_{05}}{\lambda}+\frac{A_{15}}{\lambda-1}\right) \Psi_{5}
$$


This equation possess two regular singular points, at $\lambda=0$ and 1 , and an irregular one at the point of $\infty$. We require the following conditions:

$$
\operatorname{diag}\left(A_{05}+A_{15}\right)=-\frac{\Theta_{\infty 5}}{2} \sigma_{3}, \quad \Theta_{\infty 5} \in \mathbb{C} ;
$$

and that there exists $R_{\nu 5} \in \mathrm{SL}(2, \mathbb{C})$ such that

$$
R_{\nu 5}^{-1} A_{\nu 5} R_{\nu 5}=\frac{\Theta_{\nu 5}}{2} \sigma_{3}, \quad \Theta_{\nu 5} \in \mathbb{C} \backslash \mathbb{Z}, \quad \nu=0,1 .
$$

To define the monodromy data let us define the canonical solutions $\Psi_{5}^{k}$ of Equation (3.40) by setting their asymptotics at the infinity point as

$$
\begin{gathered}
\Psi_{5}^{k}=\left(I+\sum_{m=1}^{\infty} \Psi_{m \infty 5} \lambda^{-m}\right) \exp \left(\left(\frac{\lambda t_{5}}{2}-\frac{\Theta_{\infty 5}}{2} \ln \lambda\right) \sigma_{3}\right), \\
\lambda \rightarrow \infty, \quad-\frac{3}{2} \pi+\pi k<\arg \left(\lambda t_{5}\right)<\frac{\pi}{2}+\pi k, \quad k \in \mathbb{Z} .
\end{gathered}
$$

The single-valued function $\Psi_{5}^{k}$ can be defined in the domain $\mathbb{C} \backslash\left([0,1] \cup\left[0, \infty e^{\frac{i \pi}{2}+\pi k i}\right)\right.$, where we choose the main branch of $\ln \lambda$ : $\operatorname{Im} \ln \lambda=\arg \lambda$. Using Asymptotics (3.43), one proves that

$$
\Psi_{5}^{k+2}\left(\lambda e^{2 \pi i}\right)=\Psi_{5}^{k}(\lambda) e^{-\pi i \Theta_{\infty 5} \sigma_{3}} .
$$

Now we define the Stokes matrices $S_{k}$ as

$$
\Psi_{5}^{k+1}(\lambda)=\Psi_{5}^{k}(\lambda) S_{k}
$$

Definitions (3.43) and (3.45) yield

$$
S_{2 l}=\left(\begin{array}{cc}
1 & 0 \\
s_{2 l} & 1
\end{array}\right), \quad S_{2 l+1}=\left(\begin{array}{cc}
1 & s_{2 l+1} \\
0 & 1
\end{array}\right), \quad l \in \mathbb{Z},
$$

where $s_{k}$ are called the Stokes multipliers. Using Equations (3.44) and (3.45) one finds

$$
S_{k+2}=e^{\pi i \Theta_{\infty 5} \sigma_{3}} S_{k} e^{-\pi i \Theta_{\infty 5} \sigma_{3}} .
$$

The monodromy matrix at the point of infinity, $M_{k \infty 5}$, for the $\Psi_{5}^{k}$-function is given by the equation

$$
\Psi_{5}^{k}\left(\lambda e^{-2 \pi i}\right)=\Psi_{5}^{k}(\lambda) M_{k \infty 5}
$$

Comparing Equations (3.44) and (3.45), one arrives at

$$
M_{k \infty 5}=S_{k} S_{k+1} e^{\pi i \Theta_{\infty 5} \sigma_{3}}
$$

In the following we set

$$
M_{\infty 5} \equiv M_{0 \infty 5} .
$$

All the others $M_{k \infty 5}$ can be expressed in terms of $M_{\infty 5}$ via the recurrence formula

$$
M_{k+1 \infty 5}=S_{k}^{-1} M_{k \infty 5} S_{k}
$$

and Equation (3.48). The monodromy matrices $M_{\nu 5}, \nu=0,1$, at the regular singularities $\lambda=\nu$ of Equation (3.40) are defined with the help of the paths given in Figure 7 by the same formulae (3.4), (3.5), (3.7), and (3.9) with the subscript 6 changed to 5 . 


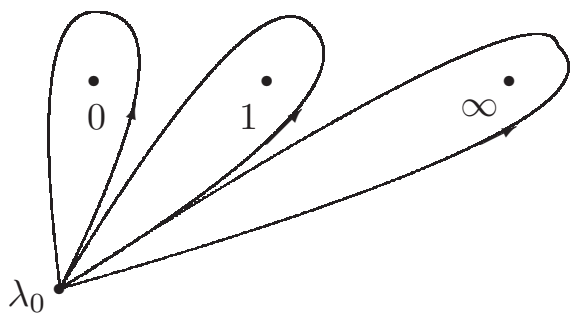

Figure 7

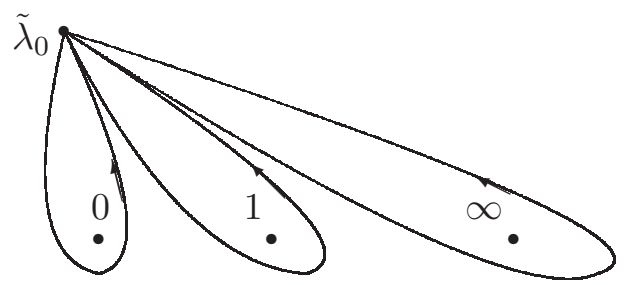

Figure 8

The cyclic relation reads

$$
M_{05} M_{15} M_{\infty 5}=I .
$$

The monodromy group $M G_{5}$ is a subgroup of $\mathrm{SL}(2, \mathbb{C})$ generated by the matrices $M_{\nu 5}$. So, under the monodromy group we mean a particular monodromy representation of the fundamental group $\pi\left(\lambda_{0}, \overline{\mathbb{C}} \backslash\{0,1, \infty\}\right)$. Together with the group $M G_{5}$ defined above we will use another one, $\widetilde{M G}$, which is generated by the monodromy matrices $\widetilde{M}_{\nu 6} \equiv \widetilde{M}_{0 \nu 6}$ obtained by the analytic continuation of the same canonical solution $\Psi_{5}^{0}(\lambda)$ but along the paths presented in Figure 8.

These matrices obey the following cyclic relation:

$$
\widetilde{M}_{\infty 5} \widetilde{M}_{15} \widetilde{M}_{05}=I
$$

The relation between both monodromy groups can be obtained by comparing representations of the fundamental groups in Figures 7 and 8 together with our way of defining a singlevalued branch of the function $\Psi_{5}^{0}$ explained in the paragraph below the asymptotics at the point of infinity (3.43):

$$
\widetilde{M}_{15}=M_{05} M_{15} M_{05}^{-1}, \quad \widetilde{M}_{05}=M_{05}, \quad \widetilde{M}_{\infty 5}=M_{\infty 5} .
$$

The sets of monodromy data $\mathcal{M}_{5}$ (or $\widetilde{\mathcal{M}}_{5}$ ) are ordered sets of the matrix elements $\left\{M_{\nu 5}\right\}_{\nu=0,1, \infty}$ (or $\left.\left\{\widetilde{M}_{\nu 5}\right\}_{\nu=0,1, \infty}\right)$ completed with three complex parameters $\Theta_{\nu 5}(\nu=$ $0,1, \infty)$ satisfying the following equations

$$
\begin{aligned}
& \nu=0,1: \quad \operatorname{tr} M_{\nu 5}=2 \cos \left(\pi \Theta_{\nu 5}\right), \quad \Theta_{\nu 5} \in \mathbb{C} \backslash \mathbb{Z}, \\
& \nu=\infty: \quad \operatorname{tr} M_{\infty 5}=2 \cos \left(\pi \Theta_{\infty 5}\right)+e^{-\pi i \Theta_{\infty 5}} s_{0} s_{1}, \quad \Theta_{\infty 5} \in \mathbb{C} .
\end{aligned}
$$

The manifold of monodromy data $\mathcal{M}_{5}\left(\Theta_{05}, \Theta_{15}, \Theta_{\infty 5}\right)$ is an algebraic variety defined by Equations (3.49), (3.51) and

$$
\operatorname{det} M_{\nu 5}=1, \quad \nu=0,1, \infty
$$

in $\mathbb{C}^{12}$ (we identify an ordered set of matrix elements $\left\{M_{\nu 5}\right\}_{\nu=0,1, \infty}$ as a point in $\mathbb{C}^{12}$ ). In an analogous way we define $\widetilde{\mathcal{M}}_{5}\left(\Theta_{05}, \Theta_{15}, \Theta_{\infty 5}\right)$. It is easy to see that the complex dimension of $\mathcal{M}_{5}\left(\Theta_{05}, \Theta_{15}, \Theta_{\infty 5}\right)$ (respectively, $\left.\widetilde{\mathcal{M}}_{5}\left(\Theta_{05}, \Theta_{15}, \Theta_{\infty 5}\right)\right)$ equals 3 .

Consider, following [30], the parametrization of $A_{\nu 5}$ :

$$
\begin{gathered}
A_{05}=\left(\begin{array}{cc}
z_{5}+\Theta_{05} / 2 & -u_{5}\left(z_{5}+\Theta_{05}\right) \\
u_{5}^{-1} z_{5} & -z_{5}-\Theta_{05} / 2
\end{array}\right) \\
A_{15}=\left(\begin{array}{cc}
-z_{5}-\left(\Theta_{05}+\Theta_{\infty 5}\right) / 2 & u_{5} y_{5}\left(z_{5}+\left(\Theta_{05}-\Theta_{15}+\Theta_{\infty 5}\right) / 2\right) \\
-\frac{1}{u_{5} y_{5}}\left(z_{5}+\left(\Theta_{05}+\Theta_{15}+\Theta_{\infty 5}\right) / 2\right) & z_{5}+\left(\Theta_{05}+\Theta_{\infty 5}\right) / 2
\end{array}\right) .
\end{gathered}
$$


We see that for fixed $t_{5}$ and formal monodromies $\Theta_{\nu 5}(\nu=0,1, \infty)$ the number of parameters $\left(u_{5}, z_{5}, y_{5}\right)$ in Equation (3.40) is 3: it exactly coincides with $\operatorname{dim} \mathcal{M}_{5}\left(\Theta_{05}, \Theta_{15}, \Theta_{\infty 5}\right)$.

The hard isomonodromy condition: $\partial_{t_{5}} \Theta_{\nu 5}=0$ and $\partial_{t_{5}} M_{\nu 5}=0$, for $\nu=0,1, \infty$ implies an additional ODE for the function $\Psi_{5}$ with respect to $t_{5}$ :

$$
\frac{d \Psi_{5}}{d t_{5}}=\left(\frac{\lambda}{2} \sigma_{3}+\frac{1}{t_{5}}\left(\frac{\Theta_{\infty 5}}{2} \sigma_{3}+A_{05}+A_{15}\right)\right) \Psi_{5}
$$

The compatibility condition of Equations (3.40) and (3.54) implies that the matrices $A_{\nu 5}$ and hence the parameters: $u_{5}, z_{5}$, and $y_{5}$, are functions of $t_{5}$. These functions are governed by the system of isomonodromy deformations,

$$
\frac{d A_{\nu 5}}{d t_{5}}=\left[\frac{1}{t_{5}}\left(\frac{\Theta_{\infty 5}}{2} \sigma_{3}+A_{05}+A_{15}\right)+\frac{\nu}{2} \sigma_{3}, A_{\nu 5}\right], \quad \nu=0,1 .
$$

In terms of the parameters $u_{5}, z_{5}$, and $y_{5}$, this system is given in [30]: eliminating the function $z_{5}$ from these equations one finds that $y_{5}\left(t_{5}\right)$ solves the fifth Painlevé equation:

$$
\begin{gathered}
\frac{d^{2} y_{5}}{d t_{5}^{2}}=\left(\frac{1}{2 y_{5}}+\frac{1}{y_{5}-1}\right)\left(\frac{d y_{5}}{d t_{5}}\right)^{2}-\frac{1}{t_{5}} \frac{d y_{5}}{d t_{5}}+\left(\frac{y_{5}-1}{t_{5}}\right)^{2}\left(\alpha_{5} y_{5}+\frac{\beta_{5}}{y_{5}}\right)+ \\
\gamma_{5} \frac{y_{5}}{t_{5}}+\delta_{5} \frac{y_{5}\left(y_{5}+1\right)}{y_{5}-1} \\
\alpha_{5}=\frac{1}{2}\left(\frac{\Theta_{05}-\Theta_{15}+\Theta_{\infty 5}}{2}\right)^{2}, \quad \beta_{5}=-\frac{1}{2}\left(\frac{\Theta_{05}-\Theta_{15}-\Theta_{\infty 5}}{2}\right)^{2}, \\
\gamma_{5}=1-\Theta_{05}-\Theta_{15}, \quad \delta_{5}=-\frac{1}{2} .
\end{gathered}
$$

There is an ambiguity in the definition of the formal monodromies $\Theta_{\nu 5} \rightarrow-\Theta_{\nu 5}, \nu=0,1$, as well as in the case for the function $\Psi_{6}$. The change $\Theta_{\nu 5} \rightarrow-\Theta_{\nu 5}$ leads to a reparametrization of the matrices $A_{\nu 5}$. Contrary to the above case for $P_{6}$ this reparametrization yields a nontrivial transformation of the solution $y_{5}$. We won't discuss it here. It is important for us that we can choose the signs of $\theta_{\nu 5}, \nu=0,1$, arbitrarily, and then use the corresponding parametrization of $A_{\nu 5}$.

The $\tau$-function for the isomonodromy deformations (3.55) is defined in 30] as follows:

$$
\frac{d}{d t_{5}} \ln \tau_{5}\left(t_{5}\right)=-\frac{1}{2} \operatorname{tr}\left(\Psi_{1 \infty 5} \sigma_{3}\right)
$$

In terms of the matrix elements Equation (3.55) reads:

$$
\begin{gathered}
\frac{d}{d t_{5}} \ln \tau_{5}\left(t_{5}\right)=\left(A_{15}\right)_{11}+\frac{1}{t_{5}}\left(A_{05}+A_{15}\right)_{21}\left(A_{05}+A_{15}\right)_{12}=-z_{5}-\frac{\Theta_{05}+\Theta_{\infty 5}}{2} \\
-\frac{1}{t_{5}}\left(z_{5}-\frac{1}{y_{5}}\left(z_{5}+\frac{\Theta_{05}+\Theta_{15}+\Theta_{\infty 5}}{2}\right)\right)\left(z_{5}+\Theta_{05}-y_{5}\left(z_{5}+\frac{\Theta_{05}-\Theta_{15}+\Theta_{\infty 5}}{2}\right)\right) .
\end{gathered}
$$

We can also rewrite Equation (3.59) in the "matrix" form:

$$
\frac{d}{d t_{5}} \ln \tau_{5}\left(t_{5}\right)=\frac{1}{t_{5}}\left(\left(\frac{\Theta_{05}}{2}\right)^{2}+\left(\frac{\Theta_{15}}{2}\right)^{2}-\left(\frac{\Theta_{\infty 5}}{2}\right)^{2}\right)+\operatorname{tr}\left(\frac{1}{t_{5}} A_{05}+\frac{\sigma_{3}}{2}\right) A_{15} .
$$


In 30] the function $\sigma_{5}\left(t_{5}\right)$ :

$$
\sigma_{5}\left(t_{5}\right)=\frac{\Theta_{05}+\Theta_{\infty 5}}{2} t_{5}+t_{5} \frac{d}{d t_{5}} \ln \tau_{5}\left(t_{5}\right) .
$$

This function satisfies a second-order ODE which is quadratic with respect to $\sigma_{5}^{\prime \prime}$ (see 30]). Differentiating (3.61) and using (3.60), (3.55), and parametrization (3.52), (3.53), we find that

$$
\frac{d \sigma_{5}}{d t_{5}}=-z_{5}
$$

In the corresponding formula (C.44) of [30] there is a misprint in the sign: this sign is important for us to establish the differentiable character of our asymptotic expansion for the function $\hat{\sigma}_{6}\left(t_{6}\right)$ (see Subsection II.4 of Section 4).

\section{Formal Limit Transitions $P_{6} \rightarrow P_{5}$}

As mentioned in the Introduction we consider here two different limits. Our scheme for the derivation of these limits consists of the following steps:

1. A formal limit passage of Equation (3.1) to Equation (3.40);

2. Finding conditions which guarantee that simultaneously with the limit passage in item 1 we have the limit passage of Equation (3.10) to Equation (3.54). Actually these conditions are additional to those found in the first step. One proves that if the asymptotic expansions found in the first step are differentiable with respect to $t_{5}$, then it is possible to define asymptotic expansions for the functions $s_{\nu 6}, \nu=1, t$, (see Equation (3.12) ) to satisfy additional conditions appearing at this step. To prove that the asymptotics we found are really differentiable, one has to use the systems of isomonodromy deformations (3.11) (or, in terms of the matrix elements, Equations (C.51), (C.52), and (C.55) of [30]) and Equation (3.55) (or (C.40) of [30]). We leave this proof to the reader and write down only the formulae for $s_{\nu 6}$.

3. Presentation of the formal limit passage in terms of the matrix elements of $A_{\nu 6}$ and $A_{\nu 5}$.

4. Presentation of the limit as (formal) asymptotics for the $P_{6^{-}}$and $\tau_{6}$-functions.

To eliminate possible confusion, let us agree to supply the parameters $\lambda$ from Equations (3.1) and (3.40) with the subscripts 6 or 5 , respectively.

I.1. The first limit passage:

$$
\begin{gathered}
\varepsilon \rightarrow+0, \quad \Theta_{16}=-\frac{1}{\varepsilon}, \quad t_{6}=\varepsilon t_{5}=\mathcal{O}(\varepsilon), \quad \lambda_{6}=\varepsilon t_{5} \lambda_{5}=o(\varepsilon), \\
\lim _{\varepsilon \rightarrow+0} R_{16}^{-1} \Psi_{6}\left(\lambda_{6}, t_{6}\right)=\Psi_{5}\left(\lambda_{5}, t_{5}\right), \\
R_{16}^{-1} A_{06} R_{16}=A_{05}+\mathcal{O}(\varepsilon), \quad R_{16}^{-1} A_{t 6} R_{16}=A_{15}+\mathcal{O}(\varepsilon) .
\end{gathered}
$$

I.2.

$$
-R_{16}^{-1} \frac{d}{d t_{5}} R_{16}=\frac{\Theta_{\infty 5}}{2 t_{5}} \sigma_{3}+\mathcal{O}(\varepsilon)
$$


where $\Theta_{\infty 5}$ appears as a parameter of the limit passage, i.e., an arbitrary complex number, its notation as one of the formal monodromies related with the fact that in derivation of Equation (4.4) we took into account relation (3.41).

I.3. In terms of the matrix elements $A_{\nu 6}$ and $A_{\nu 5}$ we can write the limit $\mathbf{I}$ in two possible ways. So for the matrix elements we get the two different formal limits I.3.a. and I.3.b.. Both limits are related via the transformation generated by the Reflection (3.22); however, the leading terms of one limit do not completely define the leading terms of the other: some further terms of the expansions are needed. So the formulae given below for the limit I.3.a. do not completely define asymptotics I.3.b.

\section{I.3.a.}

$$
\Theta_{16}=-\frac{1}{\varepsilon}, \quad \Theta_{\infty 6}+\Theta_{16}=\Theta_{\infty 5}, \quad \Theta_{06}=\Theta_{05}, \quad \Theta_{t 6}=-\Theta_{15} .
$$

In Section 3 in the paragraph following Equation (3.57), we explained that the signs in

the last two equations of (4.5) can be taken arbitrarily; however, from their choice, the following formulae are strongly depended.

$$
\begin{gathered}
z_{06}=z_{5}+\mathcal{O}(\varepsilon), \quad z_{t 6}=-z_{5}-\frac{\Theta_{05}-\Theta_{15}+\Theta_{\infty 5}}{2}+\mathcal{O}(\varepsilon), \\
z_{16}=-\varepsilon\left(z_{5}-\frac{1}{y_{5}}\left(z_{5}+\frac{\Theta_{05}+\Theta_{15}+\Theta_{\infty 5}}{2}\right)\right) \times \\
\left(z_{5}+\Theta_{05}-y_{5}\left(z_{5}+\frac{\Theta_{05}-\Theta_{15}+\Theta_{\infty 5}}{2}\right)\right)+\mathcal{O}\left(\varepsilon^{2}\right), \\
u_{06} s_{16}^{2}=\varepsilon^{2} u_{5} \frac{z_{5}+\Theta_{05}}{z_{5}}+\mathcal{O}\left(\varepsilon^{3}\right), \quad u_{t 6} s_{16}^{2}=\varepsilon^{2} y_{5} u_{5}+\mathcal{O}\left(\varepsilon^{3}\right), \\
u_{16} s_{16}^{2}=\frac{\varepsilon u_{5}}{z_{5}-\frac{1}{y_{5}}\left(z_{5}+\frac{\Theta_{05}+\Theta_{15}+\Theta_{\infty 5}}{2}\right)}+\mathcal{O}\left(\varepsilon^{2}\right) .
\end{gathered}
$$

As follows from Equation (4.4) the function $s_{16}$ must satisfy the equation

$$
\frac{d}{d t_{5}} \ln s_{16}=\frac{\Theta_{\infty 5}}{2 t_{5}}+\mathcal{O}(\varepsilon)
$$


I.3.b.

$$
\begin{gathered}
\Theta_{16}=-\frac{1}{\varepsilon}, \quad \Theta_{16}-\Theta_{\infty 6}=\Theta_{\infty 5}, \quad \Theta_{06}=\Theta_{05}, \quad \Theta_{t 6}=-\Theta_{15}, \\
z_{06}=-z_{5}-\Theta_{05}+\mathcal{O}(\varepsilon), \quad z_{t 6}=z_{5}+\frac{\Theta_{05}+\Theta_{15}+\Theta_{\infty 5}}{2}+\mathcal{O}(\varepsilon), \\
z_{16}=\frac{1}{\varepsilon}+\varepsilon\left(z_{5}-\frac{1}{y_{5}}\left(z_{5}+\frac{\Theta_{05}+\Theta_{15}+\Theta_{\infty 5}}{2}\right)\right) \times \\
\left(z_{5}+\Theta_{05}-y_{5}\left(z_{5}+\frac{\Theta_{05}-\Theta_{15}+\Theta_{\infty 5}}{2}\right)\right)+\mathcal{O}\left(\varepsilon^{2}\right), \\
\frac{u_{16}}{u_{t 6}}=\varepsilon y_{5}\left(z_{5}-\frac{1}{y_{5}}\left(z_{5}+\frac{\Theta_{05}+\Theta_{15}+\Theta_{\infty 5}}{2}\right)\right)+\mathcal{O}\left(\varepsilon^{2}\right), \\
\frac{u_{16}}{u_{06}}=\varepsilon \frac{z_{5}+\Theta_{05}}{z_{5}}\left(z_{5}-\frac{1}{y_{5}}\left(z_{5}+\frac{\Theta_{05}+\Theta_{15}+\Theta_{\infty 5}}{2}\right)\right)+\mathcal{O}\left(\varepsilon^{2}\right), \\
u_{16} s_{16}^{2}=-\frac{\varepsilon u_{5}}{z_{5}-\frac{1}{y_{5}}\left(z_{5}+\frac{\Theta_{05}+\Theta_{15}+\Theta_{\infty 5}}{2}+\mathcal{O}\left(\varepsilon^{2}\right)\right.} \\
\frac{d}{d t_{5}} \ln s_{16}=\frac{d}{d t_{5}} \ln \frac{u_{5}}{z_{5}-\frac{1}{y_{5}}\left(z_{5}+\frac{\Theta_{05}+\Theta_{15}+\Theta_{\infty 5}}{2}\right)}-\frac{\Theta_{\infty 5}}{2 t_{5}}+\mathcal{O}(\varepsilon) .
\end{gathered}
$$

I.4. Substituting Asymptotics (4.3) and (4.1) into Equation (3.18) and using definition (3.60) one finds that

$$
\frac{d}{d t_{5}} \ln \tau_{6}\left(t_{6}\right)=\frac{1}{t_{5}}\left(\left(\frac{\Theta_{\infty 5}}{2}\right)^{2}-\left(\frac{\Theta_{05}}{2}\right)^{2}-\left(\frac{\Theta_{15}}{2}\right)^{2}\right)+\frac{d}{d t_{5}} \ln \tau_{5}\left(t_{5}\right)+\mathcal{O}(\varepsilon) .
$$

Substituting Asymptotics (4.6) and (4.7) into Equation (3.15) we find for the limit I.3.a.

$$
y_{6}\left(t_{6}\right)=\frac{\varepsilon t_{5}}{1+y_{5}\left(1-\frac{\Theta_{05}+\Theta_{15}-\Theta_{\infty 5}}{2\left(z_{5}+\Theta_{\infty 5}\right)}\right)}+\mathcal{O}\left(\varepsilon^{2}\right) .
$$

In case I.3.b. Equations (4.12), (4.14) and (4.15) yield

$$
y_{6}\left(t_{6}\right)=\frac{\varepsilon t_{5}}{1+\frac{1}{y_{5}}\left(1+\frac{\Theta_{05}+\Theta_{15}+\Theta_{\infty 5}}{2 z_{5}}\right)}+\mathcal{O}\left(\varepsilon^{2}\right) .
$$

Now we consider the second formal limit.

II.1.

$$
\begin{gathered}
\varepsilon \rightarrow+0, \quad \Theta_{t 6}=-\frac{1}{\varepsilon}, \quad t_{6}=\varepsilon t_{5}=\mathcal{O}(\varepsilon), \quad \lambda_{6}=\frac{1}{\lambda_{5}}, \quad \frac{\varepsilon}{\lambda_{6}^{3}}=o(1), \\
\lim _{\varepsilon \rightarrow+0} R_{t 6}^{-1} \Psi_{6}\left(\lambda_{6}, t_{6}\right)=\Psi_{5}\left(\lambda_{5}, t_{5}\right) \\
R_{t 6}^{-1} \frac{\Theta_{\infty 6}}{2} \sigma_{3} R_{t 6}=A_{05}+\mathcal{O}(\varepsilon), \quad R_{t 6}^{-1} A_{16} R_{t 6}=A_{15}+\mathcal{O}(\varepsilon)
\end{gathered}
$$

II.2.

$$
-R_{t 6}^{-1} \frac{d}{d t_{5}} R_{t 6}=\frac{1}{t_{5}}\left(\frac{\Theta_{\infty 5}}{2} \sigma_{3}+A_{05}+A_{15}\right)+\mathcal{O}(\varepsilon)
$$

where $\Theta_{\infty 5}$ is, as for the first limit, a parameter of the limit passage satisfying Equation (3.41). 
II.3.

$$
\Theta_{t 6}=-\frac{1}{\varepsilon}, \quad \Theta_{t 6}+\Theta_{06}=\Theta_{\infty 5}, \quad \Theta_{\infty 6}=\Theta_{05}, \quad \Theta_{16}=\Theta_{15} .
$$

Together with the case $\Theta_{t 6}+\Theta_{06}=\Theta_{\infty 5}$ we can consider another one: $\Theta_{t 6}-\Theta_{06}=$ $\Theta_{\infty 5}$. As explained in Section 3. transformation $\Theta_{06} \rightarrow-\Theta_{06}$ simply means that the reparametrization of the matrix $A_{06}$, which does no effect on both the $\tau_{6^{-}}$and the $P_{6^{-}}$ functions. Thus, contrary to the first limit, it is not worthwhile to consider separately these possibilities:

$$
\begin{gathered}
z_{t 6}=-\frac{z_{5}}{\varepsilon \Theta_{05}}+\mathcal{O}(1), \quad z_{06}=\frac{z_{5}}{\varepsilon \Theta_{05}}+\mathcal{O}(1), \\
z_{16}+\Theta_{16}=\left(\frac{z_{5}\left(1-y_{5}\right)}{\Theta_{05}}+1\right)\left(\Theta_{15}+\frac{1-y_{5}}{y_{5}}\left(z_{5}+\frac{\Theta_{05}+\Theta_{15}+\Theta_{\infty 5}}{2}\right)\right)+\mathcal{O}(\varepsilon), \\
u_{t 6}=-\varepsilon^{2} \frac{\Theta_{05} u_{5}}{z_{5} s_{t 6}^{2}}+\mathcal{O}\left(\varepsilon^{3}\right), \quad u_{06}=u_{t 6}+\mathcal{O}\left(\varepsilon^{3}\right), \\
u_{16}=u_{t 6} \frac{\Theta_{15}+\left(\frac{1}{y_{5}}-1\right)\left(z_{5}+\frac{\Theta_{05}+\Theta_{15}+\Theta_{\infty 5}}{2}\right)}{\Theta_{15}+\left(\frac{1}{y_{5}}-1\right)\left(z_{5}+\frac{\Theta_{05}+\Theta_{15}+\Theta_{\infty 5}}{2}\right)\left(1+\frac{\Theta_{05}}{z_{5}\left(1-y_{5}\right)}\right)}+\mathcal{O}\left(\varepsilon^{3}\right), \\
\frac{d}{d t_{5}} \ln s_{t 6}=\frac{z_{5}}{\Theta_{05}} \frac{d}{d t_{5}} \ln u_{t 6}+\mathcal{O}(\varepsilon), \\
t_{5} \frac{d}{d t_{5}} \ln u_{t 6}=-\frac{\Theta_{05}}{z_{5}}\left(z_{5}-\frac{1}{y_{5}}\left(z_{5}+\frac{\Theta_{05}+\Theta_{15}+\Theta_{\infty 5}}{2}\right)\right)+\mathcal{O}(\varepsilon), \\
t_{5} \frac{d}{d t_{5}} \ln \left(u_{t 6} z_{t 6} s_{t 6}\right)=-\left(z_{5}+\Theta_{05}-y_{5}\left(z_{5}+\frac{\Theta_{05}-\Theta_{15}+\Theta_{\infty 5}}{2}\right)\right)+\mathcal{O}(\varepsilon) .
\end{gathered}
$$

Equations (4.31) and (4.32) play a more important role in the proof of the consistency of the second limit with the isomonodromy condition (4.24) than the analogous formulae for the first limit, that is why they are written here explicitly. Note that $\Theta_{05} \neq 0$ due to the third equation in (4.25) and the condition $\Theta_{\infty 66} \neq 0$ which is imposed in Section 3 ,

II.4. Substituting Equations (4.21) and (4.23) into (3.18) and using normalization (3.2) we find the following asymptotics for the $\tau_{6}$-function:

$$
\frac{d}{d t_{5}} \ln \tau_{6}\left(t_{6}\right)=-\frac{1}{2 \varepsilon^{2} t_{5}}-\frac{\Theta_{\infty 5}}{2 \varepsilon t_{5}}+\mathcal{O}(1)
$$

Thus, the most interesting term of the asymptotic expansion (4.33), that is $\mathcal{O}(1)$, cannot be obtained directly from our result: it requires more precise expansions in (4.26) (up to $\mathcal{O}(\varepsilon)$ ). Nevertheless, there is the following trick to overcome this difficulty. Using Equations (3.18), (3.19), (3.11), and (3.2), we, following [30, find

$$
\frac{d \hat{\sigma}_{6}\left(t_{6}\right)}{d t_{6}}=-\operatorname{tr}\left(\frac{\Theta_{\infty 6}}{2} \sigma_{3} A_{t 6}\right)-\operatorname{tr} A_{t 6}^{2} .
$$

Now Equations (4.23) yield

$$
t_{6} \frac{d \hat{\sigma}_{6}\left(t_{6}\right)}{d t_{6}}=\frac{t_{5}}{2} \operatorname{tr}\left(A_{05} \sigma_{3}\right)-\frac{t_{5}}{2 \varepsilon}+\mathcal{O}(\varepsilon) .
$$

Applying now (3.41) one obtains

$$
t_{6} \frac{d \hat{\sigma}_{6}\left(t_{6}\right)}{d t_{6}}=-\frac{t_{5}}{2}\left(\frac{1}{\varepsilon}+\Theta_{\infty 5}+\operatorname{tr}\left(A_{15} \sigma_{3}\right)\right)+\mathcal{O}(\varepsilon) .
$$


Now from (C.59) or directly from Equation (4.34) we find that

$$
\begin{gathered}
t_{6} \frac{d \hat{\sigma}_{6}\left(t_{6}\right)}{d t_{6}}-\hat{\sigma}_{6}\left(t_{6}\right)=\operatorname{tr} A_{06} A_{t 6}=\frac{1}{2}\left(\operatorname{tr}\left(\left(A_{06}+A_{t 6}\right)^{2}-\operatorname{tr}\left(A_{06}\right)^{2}-\operatorname{tr}\left(A_{t 6}\right)^{2}\right)\right. \\
=\frac{1}{2}\left(\operatorname{tr}\left(\frac{\Theta_{\infty 6}}{2} \sigma_{3}+A_{16}\right)^{2}-\operatorname{tr}\left(A_{06}\right)^{2}-\operatorname{tr}\left(A_{t 6}\right)^{2}\right) \\
=\frac{\Theta_{\infty 6}}{2} \operatorname{tr}\left(A_{16} \sigma_{3}\right)+\frac{1}{2}\left(\operatorname{tr}\left(\frac{\Theta_{\infty 6}}{2} \sigma_{3}\right)^{2}+\operatorname{tr}\left(A_{16}\right)^{2}-\operatorname{tr}\left(A_{06}\right)^{2}-\operatorname{tr}\left(A_{t 6}\right)^{2}\right) .
\end{gathered}
$$

Substituting into the latter formula Equations (4.23) and (4.36) and using definitions (3.19) and (3.60) we obtain

$$
\begin{gathered}
\frac{d}{d t_{5}} \ln \tau_{6}\left(t_{6}\right)=\left(\frac{1}{t_{5}}+\varepsilon+\varepsilon^{2} t_{5}\right)\left(-\frac{1}{2 \varepsilon^{2}}-\frac{\Theta_{\infty 5}-t_{5}}{2 \varepsilon}+t_{5} \operatorname{tr}\left(A_{15}\left(\frac{1}{t_{5}} A_{05}+\frac{\sigma_{3}}{2}\right)\right)+\frac{\Theta_{\infty 5} t_{5}}{2}\right. \\
\left.+\frac{\Theta_{05}^{2}+\Theta_{15}^{2}-\Theta_{\infty 5}^{2}}{2}\right)+\mathcal{O}(\varepsilon)=-\frac{1}{2 \varepsilon^{2} t_{5}}-\frac{\Theta_{\infty 5}}{2 \varepsilon t_{5}}+\frac{d}{d t_{5}} \ln \tau_{5}\left(t_{5}\right)+\mathcal{O}(\varepsilon) .
\end{gathered}
$$

The second bracket on the r.h.s. of Equation (4.37) is nothing but the asymptotic expansion (up to the order $\mathcal{O}(\varepsilon)$ for $-\hat{\sigma}_{6}\left(t_{6}\right)$. Differentiating it with $t_{5}$ and using Equation (3.62) we find exactly Equation (4.36). In an analogous manner the differentiable character of the asymptotic expansions for $u_{\nu 6}$ and $z_{\nu 6}$ can be proved.

Inserting into the first Equation (3.15) asymptotics (4.27) - (4.29) we find that

$$
y_{6}\left(t_{6}\right)=\frac{1+\mathcal{O}(\varepsilon)}{1+\frac{y_{5}-1}{t_{5}}\left(z_{5}+\frac{\Theta_{05}-\Theta_{15}+\Theta_{\infty 5}}{2}-\frac{1}{y_{5}}\left(z_{5}+\frac{\Theta_{05}+\Theta_{15}+\Theta_{\infty 5}}{2}\right)\right)} .
$$

\section{The First Limit}

In this section we study the situation when the $\Psi_{6}$ function in the neighborhood of the isomonodromic cluster of two regular singularities is described via the $\Psi_{5}$-function, i.e., the function with the irregular singular point. First of all let's define this cluster keeping in mind the formulae (4.1) - 4.10) corresponding to the first limit I.3.a. We won't consider the limit I.3.b., as it can be obtained via the transformation (3.22).

Let us begin with the precise setting. We are considering the $\Psi_{6}\left(\lambda_{6}, t_{6}\right)$ function defined as in Section 3. We assume that its manifold of monodromy data

$$
\mathcal{M}_{6}\left(\Theta_{06}, \Theta_{16}, \Theta_{t 6}, \Theta_{\infty 6}\right)
$$

is given. It is also assumed that

$$
\begin{gathered}
\Theta_{\nu 6} \in \mathbb{C} \backslash \mathbb{Z}, \quad \nu=0,1, t, \infty \\
\Theta_{\infty 5}=\Theta_{06}+\Theta_{\infty 6} \in \mathbb{C} .
\end{gathered}
$$

The function $\Psi_{6}\left(\lambda_{6}, t_{6}, n\right)$ is a GSD of $\Psi_{6}\left(\lambda_{6}, t_{6}\right)$ defined by the following recurrence procedure:

$$
\begin{gathered}
\Psi_{6}\left(\lambda_{6}, t_{6}, 0\right)=\Psi_{6}\left(\lambda_{6}, t_{6}\right), \\
\Psi_{6}\left(\lambda_{6}, t_{6}, n+1\right)=\mathcal{L}_{1 \infty}^{-+} \Psi_{6}\left(\lambda_{6}, t_{6}, n\right), \quad n \in \mathbb{Z}_{+}=\{0,1, \ldots\} .
\end{gathered}
$$


The monodromy manifold $\mathcal{M}_{6}\left(\Theta_{06}, \Theta_{16}^{n}, \Theta_{t 6}, \Theta_{\infty 6}^{n}\right)$ for $\Psi_{6}\left(\lambda_{6}, t_{6}, n\right)$ coincides with that for $\Psi_{6}\left(\lambda_{6}, t_{6}\right)$ except for the values of the two parameters, $\Theta_{16}^{n}$ and $\Theta_{\infty 6}^{n}$ :

$$
\begin{gathered}
\Theta_{16}^{n}=\Theta_{16}-2 n \equiv-\frac{1}{\varepsilon_{n}}, \quad n \in \mathbb{Z}_{+}, \\
\Theta_{16}^{n}+\Theta_{\infty 6}^{n}=\Theta_{\infty 5}
\end{gathered}
$$

Equation (5.3) is the definition of the discrete small parameter $\varepsilon=\varepsilon_{n} \rightarrow+0$, while the parameter $\Theta_{\infty 5}$ in Equation (5.4) is defined by Equation (5.2).

The main object of our investigation are the GSD's $A_{\nu 6}^{n}\left(t_{6}\right), \nu=0,1, t$. They can be defined by means of Equation (3.1):

$$
\partial_{\lambda_{6}} \Psi_{6}\left(\lambda_{6}, t_{6}, n\right) \Psi_{6}^{-1}\left(\lambda_{6}, t_{6}, n\right)=\sum_{\nu=0,1, t} \frac{A_{\nu 6}^{n}\left(t_{6}\right)}{\lambda-\nu} .
$$

An alternative (equivalent) definition of $A_{\nu 6}^{n}\left(t_{6}\right)$ (without the usage of the auxiliary object $\left.\Psi_{6}\left(\lambda_{6}, t_{6}, n\right)\right)$ can be given as the following recurrence procedure:

$$
A_{\nu 6}^{0}\left(t_{6}\right)=A_{\nu 6}\left(t_{6}\right), \quad A_{\nu 6}^{n+1}=\tilde{A}_{\nu 6}^{n}\left(t_{6}\right)
$$

where $A_{\nu 6}\left(t_{6}\right)$ is some solution of (3.11), and $\tilde{A}_{\nu 6}^{n}\left(t_{6}\right)$ is obtained via Equations (3.38) - (3.39) with $J_{1 \infty}^{-+}$by inserting $A_{\nu 6}^{n}\left(t_{6}\right)$ into the r.-h.s.'s. In fact, in Theorem 5.1 we assume that the matrices $\left\{A_{\nu 6}^{0}\left(t_{6}\right)\right\}_{\nu=0,1, t}$ correspond to the manifold of monodromy data $\mathcal{M}_{6}\left(\Theta_{06}, \Theta_{16}, \Theta_{t 6}, \Theta_{\infty 6}\right)$; thus the function $\Psi_{6}\left(\lambda_{6}, t_{6}, n\right)$ is also implicitly presented in the second definition of $A_{\nu 6}^{n}\left(t_{6}\right)$.

We interpret the formal limit transition I as asymptotics (as $n \rightarrow+\infty$ ) of the even sequences $\left\{A_{\nu 6}^{2 n}\left(\epsilon_{n} t_{5}\right\}_{\nu=0,1, t}\right.$, or as asymptotics of the corresponding sequences of their matrix elements. The asymptotic behavior of the odd sequences $\left\{A_{\nu 6}^{2 n+1}\left(\epsilon_{n} t_{5}\right\}_{\nu=0,1, t}\right.$ is given by exactly the same formulae as for the even one (see formal limit $\mathbf{I}$ in Section 4 with $\varepsilon \rightarrow \varepsilon_{n}$. The monodromy data for "odd" limit can be obtained from the monodromy data for the "even" limit by simply changing in the latter formulae $\Theta_{6} \rightarrow \Theta_{6}-1$.

Sometimes, when it does not cause any confusion, we omit subscripts/superscripts $n$. To make a difference between the initial values of the parameters $\Theta_{16}$ and $\Theta_{\infty 6}$ in Equations (5.1), (5.2) and the parameters $\Theta_{16}^{n}$ and $\Theta_{\infty 66}^{n}$, we agree to denote the pair of initial values as

$$
\Theta_{16}^{0} \equiv \Theta_{6} \in \mathbb{C} \backslash \mathbb{Z}, \quad \Theta_{\infty 6}^{0}=\Theta_{\infty 5}-\Theta_{6} \in \mathbb{C} \backslash \mathbb{Z}
$$

while, instead of $\Theta_{16}^{n}$ and $\Theta_{\infty 6}^{n}$, we write $\Theta_{16}$ and $\Theta_{\infty 6}$, respectively. Thus, hereafter in Section 5. $\Theta_{6}$ and $\Theta_{\infty 5}$ are fixed according to (5.7) and (5.2), while $\Theta_{16}$ and $\Theta_{\infty 6}$ are dependent on $n$ such that $\Theta_{16} \underset{n \rightarrow+\infty}{\rightarrow}-\infty$ and $\Theta_{\infty 6} \underset{n \rightarrow+\infty}{\rightarrow}+\infty$ and the condition (5.1) holds.

In fact the formal limit $\mathbf{I}$ contains one more parameter $f_{0} \in \mathbb{C} \backslash\{0\}$. This parameter is hidden as the constant of integration in Equation (4.10). The asymptotic expansions of the sequences under investigation

$$
A_{\nu 6}^{2 n}\left(\epsilon_{n} t_{5}\right), \quad n \in \mathbb{Z}_{+}, \quad \nu=0,1, t,
$$

don't depend on $f_{0}$. On the language of the formulae (4.6) - (4.9) this means that if $s_{16} \rightarrow s_{16} f_{0}$, then $u_{5} \rightarrow u_{5} f_{0}^{2}$. Nevertheless we include $f_{0}$ for completeness. Now, 
denoting the matrix elements of (5.8) exactly as that for $A_{\nu 6}$ in (3.12), and the matrix elements of the monodromy matrices $M_{\nu 6}$ as

$$
M_{\nu 6}=\left(\begin{array}{ll}
m_{11}^{\nu 6} & m_{12}^{\nu 6} \\
m_{21}^{\nu 6} & m_{22}^{\nu 6}
\end{array}\right)
$$

we are ready to formulate our result.

Theorem 5.1 Assume that the complex parameters $\Theta_{0} 6, \Theta_{6}, \Theta_{t 6}$ satisfy Conditions (5.1) and (5.7). Let a point $\mu_{6} \in \mathcal{M}_{6}\left(\Theta_{06}, \Theta_{6}, \Theta_{t 6}, \Theta_{\infty 5}-\Theta_{6}\right)$ and $\left\{m_{i k}^{\nu 6}\right\}_{i, k=1,2}^{\nu=0,1, t}$ be its coordinates. Suppose that the following conditions are valid:

1. $m_{21}^{16} \neq 0$;

2 .

$$
\cos \pi l \equiv=i m_{11}^{16} \sin \pi\left(\Theta_{\infty 5}-\Theta_{6}\right)+\exp \left(-\pi i\left(\Theta_{\infty 5}-\Theta_{6}\right)\right) \cos \pi \Theta_{6} \neq-1
$$

3. The inverse monodromy problem defined by the pairs $\left(\mu_{6}, t_{6}\right)$ are solvable for all $t_{6}=$ $\varepsilon_{n} t_{5}$ where $n \geq N \in \mathbb{Z}_{+}$and $\varepsilon_{n}$ is defined in Equation (5.3), and $t_{5} \in \mathbb{C} \backslash\{0\}$ with $\left|\arg t_{5}\right|<\pi-\delta$ for some $\delta>0$;

4. The point $\mu_{6}$ corresponds to the general, i.e., transcendental solution of $P_{6}{ }^{6}$.

Let $l$ be the unique solution of Equation (5.10) under the conditions:

$$
l \neq 0, \quad|\operatorname{Re} l|<1
$$

Define the parameters $\alpha, \beta, d_{0}^{2}$ :

$$
\alpha=\frac{l-\Theta_{\infty 5}}{2}, \quad \beta=-\frac{l+\Theta_{\infty 5}}{2}, \quad d_{0}^{2}=\frac{m_{21}^{16}}{2 \pi i} \Gamma(1-\alpha) \Gamma(1-\beta),
$$

where $\Gamma(\cdot)$ is the gamma function 34 .

For arbitrary $f_{0} \in \mathbb{C} \backslash\{0\}$ define the matrix

$$
K=-f_{0}^{\sigma_{3}} e^{-\frac{\pi i}{2} \Theta_{6} \sigma_{3}}\left(\begin{array}{cc}
1 & \frac{\pi}{\Gamma(\alpha) \Gamma(\beta) \sin \pi\left(\Theta_{\infty 5}-\Theta_{6}\right)} \\
0 & 1
\end{array}\right) d_{0}^{\sigma_{3}},
$$

and consider the following equations:

$$
\begin{gathered}
\Theta_{05}=\Theta_{06}, \quad \Theta_{15}=-\Theta_{t 6}, \quad \Theta_{\infty 5}=\Theta_{16}+\Theta_{\infty 6}=-(\alpha+\beta), \\
\tilde{M}_{05}=K M_{06} K^{-1}, \quad \tilde{M}_{15}=K M_{t 6} K^{-1}, \quad \tilde{M}_{\infty 5}=K M_{\infty 6} M_{16} K^{-1},
\end{gathered}
$$

as defining the point $\tilde{\mu}_{5} \in \tilde{\mathcal{M}}_{5}\left(\Theta_{05}, \Theta_{15}, \Theta_{\infty 5}\right)$. Suppose that the inverse monodromy problem for $\left(\tilde{\mu}_{5}, t_{5}\right)$ is solvable and the functions $u_{5}=u_{5}\left(\tilde{\mu}_{5}, t_{5}\right), y_{5}=y_{5}\left(\tilde{\mu}_{5}, t_{5}\right)$, and $z_{5}=z_{5}\left(\tilde{\mu}_{5}, t_{5}\right)$ represent this solution.

Then it is possible to construct the sequences (5.8), corresponding to the given manifold $\mathcal{M}_{6}\left(\Theta_{06}, \Theta_{6}, \Theta_{t 6}, \Theta_{\infty 5}-\Theta_{6}\right)$. The formulae (4.5)-(4.10) are asymptotic expansions as $n \rightarrow+\infty$ of the matrix elements of (5.8) if:

1. The functions $u_{5}, y_{5}$, and $z_{5}$ are identified with the solution of the inverse monodromy

\footnotetext{
${ }^{6}$ We mean the $P_{6}$-transcendent, i.e., any solution which cannot be constructed in terms of the logarithmic derivatives of the hypergeometric functions.
} 
problem for $\left(\tilde{\mu}_{5}, t_{5}\right)$;

2. Equation (4.10) is supplemented with

$$
s_{16}=\varepsilon f_{0} d_{0}\left(\varepsilon t_{5}\right)^{\frac{\Theta_{\infty}}{2}}(1+\mathcal{O}(\varepsilon))
$$

3. $\varepsilon=\varepsilon_{n}$ is substituted into (4.5) (4.10) and (5.16).

Remark 5.1 The complex number $l$ is defined by Conditions (5.10) and (5.11) up to a sign, which means simply the permutation $\alpha \leftrightarrow \beta$. This permutation doesn't influence the asymptotics that we study, as well as the indefiniteness of the sign $d_{0}$ in (5.12).

Remark 5.2 Some of the conditions imposed on $\mu_{6}$ (see Conditions 1, 2, 4) are not necessary for the possibility to interpret the formal limit as an asymptotic expansion; however, their violation requires special investigation. In particular, we can apply this theorem not only to the $P_{6}$ transcendent, but also for most of the solutions that can be constructed via the classical special functions.

Remark 5.3 To find the Stokes multipliers for the limiting $P_{5}$ equation one can use Equation (3.48).

Derivation. Here I outline only the calculational scheme for the solution of the direct and inverse monodromy problem, while an explanation of how such calculations work for the justification of the asymptotic expansions, the reader will find in Section [ in the derivation of Theorem 6.1 Although the derivations are different, the scheme of justification is the same and can be based on the work 35.

For some $\delta_{\text {in }}, \delta_{\text {out }}$ such that $\frac{1}{3}<\delta_{\text {in }}<\delta_{\text {out }}<1$ and for all rather small $\varepsilon>0$ we can present the $\lambda_{6}$-complex plane as $\mathbb{C}=\Omega_{\text {in }}(\varepsilon) \cup \Omega_{\text {out }}(\varepsilon)$, where

$$
\lambda_{6} \in \Omega_{\text {in }}(\varepsilon) \Leftrightarrow\left|\lambda_{6}\right| \leq \mathcal{O}\left(\varepsilon^{\frac{1}{2}+\frac{\delta_{\text {in }}}{2}}\right), \quad \lambda_{6} \in \Omega_{\text {out }}(\varepsilon) \Leftrightarrow\left|\lambda_{6}\right| \geq \mathcal{O}\left(\varepsilon^{\frac{1}{2}+\frac{\delta_{\text {out }}}{2}}\right) .
$$

Thus the matching domain, $\Omega_{\text {mat }}(\varepsilon)=\Omega_{\text {in }}(\varepsilon) \cap \Omega_{\text {out }}(\varepsilon)$, is nonempty for all rather small $\varepsilon$.

In the cluster domain we approximate the function $\Psi_{6}=\Psi_{6}\left(\lambda_{6}, t_{6}, n\right)$ as follows

$$
\Psi_{6} \underset{\varepsilon \rightarrow 0}{=} R_{16}\left(1+\mathcal{O}\left(\varepsilon^{\delta}\right)\right) \Psi_{5}^{0} K, \quad \lambda_{6} \in \Omega_{i n}(\varepsilon), \quad \delta>0,
$$

where $\Psi_{5}^{0}=\Psi_{5}^{0}\left(\lambda_{5}, t_{5}\right)$ is the canonical solution of Equation (3.40) with monodromy data to be determined, as well as the constant matrix $K \in \mathrm{SL}(2, \mathbb{C})$. Using Approximation (5.17) one finds Equations (4.5) - (4.10), (5.15), and the first two equations in (5.14). We have to prove (5.13), the last equation in (5.14) and (5.16), as well as for the justification, it is important to establish the following

$$
K=\lim _{\substack{\varepsilon \rightarrow 0 \\ \lambda_{0} \in \Omega_{\text {mat }}(\varepsilon)}}\left(R_{16}\right)\left(\Psi_{5}^{0}\right)^{-1} \Psi_{\text {out }},
$$

where $\Psi_{\text {out }}$ is an approximation for $\Psi_{6}$ in the domain $\Omega_{\text {out }}(\varepsilon)$ :

$$
\Psi_{6}=\left(I+\mathcal{O}\left(\varepsilon^{\delta}\right)\right) \Psi_{\text {out }}, \quad \lambda_{6} \in \Omega_{\text {out }}(\varepsilon), \quad \delta>0 .
$$


We construct $\Psi_{\text {out }}$ by making use of the function $Y(x ; \alpha, \beta, \gamma)$ constructed by Jimbo [1]. While constructing $\Psi_{\text {out }}$ we find the conditions (5.10) - (5.12) and $m_{21}^{16} \neq 0$. Since the function $Y(x ; \alpha, \beta, \gamma)$ plays an important role not only in the present derivation but in the one in Section [6] we, following [1], recall its basic properties:

$$
\begin{gathered}
Y(x) \equiv Y(x ; \alpha, \beta, \gamma) x^{\frac{\gamma-1}{2}}(x-1)^{\frac{\alpha+\beta+1-\gamma}{2}} \\
=\left(\begin{array}{c}
F\left(\alpha, \alpha+1-\gamma, \alpha-\beta ; \frac{1}{x}, \frac{\beta(\beta+1-\gamma)}{(\beta-\alpha)(\beta-\alpha+1) x} F\left(\beta, \beta+2-\gamma, \beta-\alpha+2 ; \frac{1}{x}\right)\right. \\
\frac{\alpha(\alpha+1-\gamma)}{(\alpha-\beta)(\alpha-\beta+1) x} F\left(\alpha+1, \alpha+2-\gamma, \alpha-\beta+2 ; \frac{1}{x}\right), F\left(\beta, \beta+1-\gamma, \beta-\alpha ; \frac{1}{x}\right)
\end{array}\right) \\
\times x^{\frac{\beta-\alpha}{2} \sigma_{3}} x^{\frac{\gamma-1-\alpha-\beta}{2}}(x-1)^{\frac{\alpha+\beta+1-\gamma}{2}} \\
\Theta_{\infty Y} \equiv \alpha-\beta, \Theta_{0 Y} \equiv 1-\gamma, \Theta_{1 Y} \equiv-\alpha-\beta-1+\gamma \in \mathbb{C} \backslash \mathbb{Z}
\end{gathered}
$$

where $F(\cdot, \cdot, \cdot ; \cdot)$ denotes the Gauss hypergeometric function 34 .

$$
Y(x) \underset{x \rightarrow \nu=0,1}{=} G_{\alpha \beta \gamma}^{\nu}(I+\mathcal{O}(x-\nu))(x-\nu)^{\frac{\Theta_{\nu Y}}{2} \sigma_{3}} C_{\alpha \beta \gamma}^{\nu} \underset{x \rightarrow \infty}{=}\left(I+\mathcal{O}\left(x^{-1}\right)\right) x^{\frac{\beta-\alpha}{2} \sigma_{3}},
$$

where

$$
\begin{aligned}
& G_{\alpha \beta \gamma}^{0}=\frac{1}{\beta-\alpha}\left(\begin{array}{cc}
\beta+1-\gamma & \beta \\
\alpha+1-\gamma & \alpha
\end{array}\right), \quad G_{\alpha \beta \gamma}^{1}=\frac{1}{\beta-\alpha}\left(\begin{array}{cc}
1 & \beta(\beta+1-\gamma) \\
1 & \alpha(\alpha+1-\gamma)
\end{array}\right), \\
& C_{\alpha \beta \gamma}^{0}=\left(\begin{array}{cc}
e^{-\pi i(\alpha+1-\gamma) \frac{\Gamma(\gamma-1) \Gamma(\alpha-\beta+1)}{\Gamma(\gamma-\beta) \Gamma(\alpha)}} & -e^{-\pi i(\beta+1-\gamma) \frac{\Gamma(\gamma-1) \Gamma(\beta-\alpha+1)}{\Gamma(\gamma-\alpha) \Gamma(\beta)}} \\
e^{-\pi i \alpha \frac{\Gamma(1-\gamma) \Gamma(\alpha-\beta+1)}{\Gamma(1-\beta) \Gamma(\alpha+1-\gamma)}} & -e^{-\pi i \beta} \frac{\Gamma(1-\gamma) \Gamma(\beta-\alpha+1)}{\Gamma(1-\alpha) \Gamma(\beta+1-\gamma)}
\end{array}\right), \\
& C_{\alpha \beta \gamma}^{1}=\left(\begin{array}{cc}
-\frac{\Gamma(\alpha+\beta+1-\gamma) \Gamma(\alpha-\beta+1)}{\Gamma(\alpha+1-\gamma) \Gamma(\alpha)} & \frac{\Gamma(\alpha+\beta+1-\gamma) \Gamma(\beta-\alpha+1)}{\Gamma(\beta+1-\gamma) \Gamma(\beta)} \\
-e^{-\pi i\left(\gamma-\alpha-\beta-1 \frac{\Gamma(\gamma-\alpha-\beta-1) \Gamma(\alpha-\beta+1)}{\Gamma(1-\beta) \Gamma(\gamma-\beta)}\right.} & e^{-\pi i(\gamma-\alpha-\beta-1) \frac{\Gamma(\gamma-\alpha-\beta-1) \Gamma(\beta-\alpha+1)}{\Gamma(1-\alpha) \Gamma(\gamma-\alpha)}}
\end{array}\right), \\
& \frac{d Y(x)}{d x}=\left(\frac{A_{0 Y}}{x}+\frac{A_{1 Y}}{x-1}\right) Y(x), \quad A_{0 Y}+A_{1 Y}=\frac{\beta-\alpha}{2} \sigma_{3}, \\
& A_{0 Y}=\frac{1}{\beta-\alpha}\left(\begin{array}{cc}
-\frac{(\alpha+\beta)(1-\gamma)+2 \alpha \beta}{2} & \beta(\beta+1-\gamma) \\
-\alpha(\alpha+1-\gamma) & \frac{(\alpha+\beta)(1-\gamma)+2 \alpha \beta}{2}
\end{array}\right) \text {, } \\
& A_{1 Y}=\frac{1}{\beta-\alpha}\left(\begin{array}{cc}
-\frac{(\alpha+\beta)(1-\gamma)+\alpha^{2}+\beta^{2}}{2} & -\beta(\beta+1-\gamma) \\
-\alpha(\alpha+1-\gamma) & -\frac{(\alpha+\beta)(1-\gamma)+\alpha^{2}+\beta^{2}}{2}
\end{array}\right) \text {. }
\end{aligned}
$$

To the above-mentioned properties of $Y(x)$ pointed out by Jimbo, we add up the following one, which is important in the derivation of our main results in this and the next section:

$$
\begin{gathered}
Y(x) \underset{x \in \underline{\overline{5.31}}}{\frac{1}{\sqrt{\alpha-\beta}}}\left(\begin{array}{ll}
1 & \beta \\
1 & \alpha
\end{array}\right)\left(I+\mathcal{O}\left(x^{\tilde{\delta}}\right)\right) \exp \left(\left(\frac{1-\gamma}{2 x}+\frac{\alpha+\beta}{2} \ln \frac{1-\gamma}{x}\right) \sigma_{3}\right) \hat{K}(\varkappa), \\
\hat{K}(\varkappa)=\left(I+\mathcal{O}\left(x^{\tilde{\delta}}\right)\right) \sqrt{\alpha-\beta} \sigma_{3}\left(\begin{array}{cc}
\frac{\Gamma(\alpha-\beta)}{\Gamma(\alpha)} & \frac{\Gamma(\beta-\alpha)}{\Gamma(\beta)} \\
-e^{i \pi \alpha \varkappa \frac{\Gamma(1+\alpha-\beta)}{\Gamma(1-\beta)}} & e^{i \pi \beta \varkappa \Gamma(1+\beta-\alpha)}
\end{array}\right)(1-\gamma)^{\frac{\beta-\alpha}{2} \sigma_{3}},
\end{gathered}
$$




$$
\left|\frac{1-\gamma}{x^{2}}\right| \underset{\varepsilon \rightarrow+0}{=} \mathcal{O}\left(x^{\tilde{\delta}}\right), \quad\left|\frac{1-\gamma}{x}\right| \underset{\varepsilon \rightarrow+0}{=}+\infty, \quad-\pi+\frac{\varkappa \pi}{2}<\arg \frac{1-\gamma}{x}<\pi+\frac{\varkappa \pi}{2}, \quad \varkappa= \pm 1 .
$$

In Equation (5.29), and thereafter, the notation $\mathcal{O}\left(x^{\tilde{\delta}}\right)$ means that the corresponding estimate holds for some $\tilde{\delta}>0$. The precise (the largest possible) value of $\tilde{\delta}$ in such estimates are not important in our scheme of derivation.

For a proof of Asymptotics (5.29) we need a special asymptotic expansion for the Gauss hypergeometric function $F(a, b, c ; x)$ which can be found in 34]:

$$
\begin{gathered}
F(a, b, c ; z)=e^{i \pi a \varkappa} \frac{\Gamma(c)}{\Gamma(c-a)}(b z)^{-a}\left(1+\mathcal{O}\left(|b z|^{-1}\right)\right)+\frac{\Gamma(c)}{\Gamma(a)} e^{b z}(b z)^{a-c}\left(1+\mathcal{O}\left(|b z|^{-1}\right)\right), \\
0<|z|<1, \quad|b z| \rightarrow \infty, \quad-\pi+\frac{\varkappa \pi}{2}<\arg (b z)<\pi+\frac{\varkappa \pi}{2}, \quad \varkappa= \pm 1 .
\end{gathered}
$$

In the domain (5.31) one finds:

$$
x^{\frac{\beta-\alpha}{2} \sigma_{3}} x^{\frac{\gamma-1-\alpha-\beta}{2}}(x-1)^{\frac{\alpha+\beta+1-\gamma}{2}}=x^{\frac{\beta-\alpha}{2} \sigma_{3}} e^{-\frac{\alpha+\beta+1-\gamma}{2 x}}\left(1+\mathcal{O}\left(x^{\tilde{\delta}}\right)\right) .
$$

Substituting Expansions (5.32) and (5.33) into Equation (5.20) one arrives at Equations (5.29)-(5.31).

We construct the function $\Psi_{\text {out }}$ as follows:

$$
\begin{gathered}
\Psi_{\text {out }}\left(\lambda_{6}\right)=G^{-1} Y(x) C_{0}^{-1}, \quad x=\frac{1}{\lambda_{6}}, \\
G=G_{\alpha \beta \gamma}^{0}(\beta-\alpha)(1-\gamma)^{-\frac{1}{2}} d_{0}^{\sigma_{3}}(1-\gamma)^{\frac{\alpha+\beta-1}{2} \sigma_{3}}, \\
C_{0}=e^{\frac{\pi i}{2}(1-\gamma+\alpha+\beta)}(1-\gamma)^{\frac{1}{2}}(\beta-\alpha)^{-1}(1-\gamma)^{\frac{1-\alpha-\beta}{2} \sigma_{3}} d_{0}^{-\sigma_{3}} C_{\alpha \beta \gamma}^{0},
\end{gathered}
$$

where $\alpha$ and $\beta$, satisfying the conditions (5.21), and $d_{0} \in \mathbb{C} \backslash\{0\}$ are the parameters to be determined, and

$$
1-\gamma=\Theta_{\infty 5}+\frac{1}{\varepsilon_{n}} \rightarrow+\infty, \quad 1-\gamma=\Theta_{\infty 5}-\Theta_{6}+2 n, \quad \gamma-\alpha-\beta-1=\Theta_{6}-2 n,
$$

as it follows from Equations (5.3), (5.7), (5.14), and (5.22).

By using (5.22) and (5.29) - (5.31) we find that the function $\Psi_{\text {out }}$ has the following asymptotic behavior

$$
\begin{array}{rlrl}
\lambda_{6} \rightarrow \infty: & \Psi_{\text {out }} & \sim\left(\frac{1}{\lambda_{6}}\right)^{\frac{\Theta_{\infty} 6}{2} \sigma_{3}} \\
\lambda_{6} \rightarrow 1: & \Psi_{\text {out }} & \sim G^{-1} G_{\alpha \beta \gamma}^{1}\left(\frac{1}{\lambda_{6}}-1\right)^{\frac{\Theta_{16}}{2} \sigma_{3}} C_{\alpha \beta \gamma}^{1} C_{0}^{-1}, \\
\lambda_{6} \rightarrow 0, & \lambda_{6} \in \Omega_{\text {mat }}(\varepsilon), \quad-\pi+\frac{\varkappa \pi}{2}<\arg \lambda_{6}<\pi+\frac{\kappa \pi}{2}, \\
\Psi_{\text {out }} \sim \frac{G^{-1}}{\sqrt{\alpha-\beta}}\left(\begin{array}{cc}
1 & \beta \\
1 & \alpha
\end{array}\right) \exp \left(\left(\frac{(1-\gamma) \lambda_{6}}{2}-\frac{\alpha+\beta}{2} \ln \frac{1}{(1-\gamma) \lambda_{6}}\right) \sigma_{3}\right) \hat{K}(\varkappa) C_{0}^{-1} .
\end{array}
$$


We see from (5.37) that $\Psi_{\text {out }}$ satisfies the same normalization condition as the function $\Psi_{6}$. Thus, the monodromy matrices at $\lambda_{6}=\infty$ for both functions coincide, $M_{\infty \text { out }}=M_{\infty 6}$. Our next step will be to set the parameters $\alpha-\beta$ and $d_{0}$ such that the following equation

$$
M_{1 \text { out }}=M_{16}
$$

holds. To calculate asymptotically $M_{1 \text { out }}$ we use Expansion (5.38), Equations (5.35), (5.24) and the following formulae for the $\Gamma$-function [34]:

$$
\begin{gathered}
\frac{\Gamma(z+\mu)}{\Gamma(z+\nu)}=z^{\mu-\nu}\left(1+\mathcal{O}\left(z^{-1}\right)\right), \quad|z| \rightarrow \infty, \quad|\arg z|<\pi, \\
\Gamma(z) \Gamma(1-z)=\frac{\pi}{\sin (\pi z)} .
\end{gathered}
$$

One notices that

$$
\operatorname{det} C_{\alpha \beta \gamma}^{0}=\frac{\gamma-1}{\beta-\alpha} e^{-\pi i(\alpha+\beta+1-\gamma)},
$$

and finds

$$
\begin{gathered}
C_{0}^{-1}=(1-\gamma)^{\frac{\alpha-\beta}{2} \sigma_{3}} e^{\frac{\pi i}{2}(\alpha+\beta+1-\gamma)}\left(C+\mathcal{O}\left(x^{\tilde{\delta}}\right)\right) d_{0}^{\sigma_{3}} \\
C=\left(\begin{array}{cc}
\frac{\Gamma(\beta-\alpha+1)}{\Gamma(1-\alpha)} e^{\pi i(1-\beta)} & \frac{\Gamma(\beta-\alpha+1)}{\Gamma(\beta)} \frac{\sin \pi(\alpha+1-\gamma)}{\sin \pi(1-\gamma)} e^{-\pi i(\beta-\gamma)} \\
\frac{\Gamma(\alpha-\beta+1)}{\Gamma(1-\beta)} e^{\pi i(1-\alpha)} & \frac{\Gamma(\alpha-\beta+1)}{\Gamma(\alpha)} \frac{\sin \pi(\beta+1-\gamma)}{\sin \pi(1-\gamma)} e^{-\pi i(\alpha-\gamma)}
\end{array}\right) .
\end{gathered}
$$

Note that according to the last two equations (5.36) $C$ is the constant matrix. Using Equations (5.41) and (5.42) we obtain the leading term of asymptotics for $C_{\alpha \beta \gamma}^{1}$ (5.25):

$$
\begin{gathered}
C_{\alpha \beta \gamma}^{1} \underset{\varepsilon \rightarrow 0}{\sim}(1-\gamma)^{\frac{\alpha+\beta}{2} \sigma_{3}}\left(\begin{array}{cc}
1 & 0 \\
0 & \frac{e^{\pi i(\alpha+\beta+1-\gamma)}}{1-\gamma}
\end{array}\right) \\
\times\left(\begin{array}{cc}
-\frac{\Gamma(\alpha-\beta+1)}{\Gamma(\alpha)} & \frac{\Gamma(\beta-\alpha+1)}{\Gamma(\beta)} \\
\frac{\sin \pi(\beta+1-\gamma)}{\sin \pi(\alpha+\beta+1-\gamma)} \frac{\Gamma(\alpha-\beta+1)}{\Gamma(1-\beta)} & -\frac{\sin \pi(\alpha+1-\gamma)}{\sin \pi(\alpha+\beta+1-\gamma)} \frac{\Gamma(\beta-\alpha+1)}{\Gamma(1-\alpha)}
\end{array}\right)(1-\gamma)^{\frac{\beta-\alpha}{2} \sigma_{3} .} .
\end{gathered}
$$

Now Equations (5.43) and (5.44) yield

$$
M_{1 \text { out }}=C_{1}^{-1} e^{\pi i(\gamma-1-\alpha-\beta)} C_{1} .
$$

Hence Equation (5.40) in the notation (5.9) reads as

$$
\begin{aligned}
& m_{11}^{16}=\frac{i}{\sin \pi(1-\gamma)}\left(\cos \pi(\alpha+\beta+1-\gamma) e^{-\pi i(1-\gamma)}-\cos \pi(\alpha-\beta)\right) \\
& m_{22}^{16}=\frac{i}{\sin \pi(1-\gamma)}\left(\cos \pi(\alpha-\beta)-\cos \pi(\alpha+\beta+1-\gamma) e^{\pi i(1-\gamma)}\right) \\
& m_{21}^{16}=\frac{2 \pi i d_{0}^{2}}{\Gamma(1-\alpha) \Gamma(1-\beta)}, \quad m_{12}^{16}=-\frac{2 \pi i \sin \pi(\alpha+1-\gamma) \sin \pi(\beta+1-\gamma)}{d_{0}^{2} \Gamma(\alpha) \Gamma(\beta) \sin ^{2} \pi(1-\gamma)} .
\end{aligned}
$$

Now define $l=\alpha-\beta$ and recall (5.36) to derive from Equations (5.46) and (5.47) Formulae (5.10) and (5.12), respectively. It was supposed that $l \neq 0, \pm 1$ (see Equation (5.21)). The natural requirement $|\operatorname{Re}| l<1$ means no additional restrictions, since, thanks to the equation $\alpha+\beta=-\Theta_{\infty 5}$, the shift $l \rightarrow l+2$ leads, simply, to a redefinition of $d_{0}$; thus we get (5.11). 
Turning to the matching (5.18), we notice that $(1-\gamma) / x=\lambda_{5} t_{5}$; and by recalling asymptotics of $\Psi_{5}^{0}$ (3.43) we confirm not only the last equation (5.14), but also obtain that

$$
\begin{gathered}
R_{16}=G^{-1}\left(\begin{array}{ll}
1 & \beta \\
1 & \alpha
\end{array}\right)\left(1+\mathcal{O}\left(x^{\tilde{\delta}}\right)\right) t_{5}^{-\frac{\Theta_{\infty} 5}{2} \sigma_{3}} f_{0}^{-\sigma_{3}}, \quad \in \mathbb{C} \backslash\{0\}, \\
K=\lim _{\varepsilon \rightarrow 0} f_{0}^{\sigma_{3}} \hat{K}(-1) C_{0}^{-1} / \sqrt{\alpha-\beta} .
\end{gathered}
$$

We can further simplify Equation (5.48) with the help of Equations (5.34) and (5.23):

$$
R_{16}=d_{0}^{-\sigma_{3}}(1-\gamma)^{\frac{\Theta_{\infty} 5}{2} \sigma_{3}}\left(1+\mathcal{O}\left(x^{\tilde{\delta}}\right)\right) t_{5}^{-\frac{\Theta_{\infty} 5}{2} \sigma_{3}} f_{0}^{-\sigma_{3}} .
$$

Equation (5.50) is equivalent, up to the leading term, to Equation (5.16). Finally, substituting into (5.49) formulae (5.30), (5.36), and (5.43), we arrive at Equation (5.13).

\section{The Second Limit}

Comparing the formulae for $s_{16}$ (4.10) and (4.17) for the first limit passage with the analogous formula for $s_{t 6}$ (4.30) for the second limit, one finds the "principle" distinction between the limits: while Equations (4.10) and (4.17) are "integrable" Equation 4.30) is not. Thus it is not clear how to set the constant of integration in the definition of $s_{t 6}$. As the result asymptotics of $u_{\nu 6}, \nu=0,1, t$ is found here up to the factor $c$ : $s_{t 6} \rightarrow c s_{t 6} \Leftarrow u_{\nu 6} \rightarrow c^{-2} u_{\nu 6}$. This fact does not influence the functions $y_{6}$ and $\tau_{6}$, whose asymptotics are properly defined in the case of the second limit passage (see below Theorem 6.1). The problem of how to cope with the ambiguity of $c$, i.e., to set $s_{t 6}$ in terms of $\mathcal{M}_{6}\left(\Theta_{06}, \Theta_{16}, \Theta_{t 6}, \Theta_{\infty 6}\right)$, is left for further investigation. This explains some differences appearing in the formulations of Theorems [5.1 and 6.1] here we also omit the nonessential $f_{0}$-like parameter (see (5.13) ).

As it is mentioned in Introduction on the level of the Painlevé and the corresponding $\tau$-functions both limits are equivalent. We show that at the end of this section in Proposition 6.1 Nevertheless we present also the direct derivation because:

1. This result is easy to extend for the cluster on the arbitrary regular background, i.e., for the Garnier systems (see Corollary 6.1).

2. It is interesting to see how the "nonintegrability" of Equation (4.30) manifests itself in our asymptotic calculations which do not contain any integration in the usual sense.

Actually I started my studies with the second cluster as it naturally appeared when one inserts the expansion $\left(\lambda_{6}-t_{6}\right)^{-1}=\lambda_{6}^{-1}+t_{6} \lambda_{6}^{-2}+\ldots$ into Equation (3.1). After I realized that the above "c-problem" is not the intrinsic "cluster problem", I found the first limit, where such a " $c$-problem" does not appear. Note that applying Proposition 6.1] to Theorem 6.1 we cannot obtain the complete result for the first limit, as it stated in Theorem 5.1 .

Let us begin with the regularization of the second limit. Suppose that $\mathcal{M}_{6}\left(\Theta_{06}, \Theta_{16}, \Theta_{t 6}, \Theta_{\infty 6}\right)$ is given and define the parameters $\varepsilon_{n}, \Theta_{\infty 5}, \Theta_{6}$ as follows:

$$
\begin{gathered}
\Theta_{t 6}=\Theta_{6}-2 n \equiv-\frac{1}{\varepsilon_{n}}, \quad n \in \mathbb{Z}_{+}, \quad \Theta_{6} \in \mathbb{C} \backslash \mathbb{Z}, \\
\Theta_{t 6}+\Theta_{06}=\Theta_{\infty 5}, \quad \Theta_{\infty 5}-\Theta_{6} \in \mathbb{C} \backslash \mathbb{Z} .
\end{gathered}
$$


Here again our parameter $\varepsilon=\varepsilon_{n} \rightarrow+0$ is discrete. The main object of our investigation is the GSD $A_{\nu 6}^{k}\left(t_{6}\right) \nu=0,1, t$ and $k \in \mathbb{Z}_{+}$, which is defined via the recurrence procedure:

$$
A_{\nu 6}^{0}\left(t_{6}\right)=A_{\nu 6}\left(t_{6}\right), \quad A_{\nu 6}^{k+1}\left(t_{6}\right)=\tilde{A}_{\nu 6}^{k}\left(t_{6}\right),
$$

where $A_{\nu 6}\left(t_{6}\right)$ is the solution of System (3.11) corresponding to $\mathcal{M}_{6}\left(\Theta_{06}, \Theta_{16}, \Theta_{t 6}, \Theta_{\infty 6}\right)$, and $\tilde{A}_{\nu 6}^{k}\left(t_{6}\right)$ is obtained via (3.32) (3.35) (with $J_{0 t}^{+-}$) by inserting $A_{\nu 6}^{k}\left(t_{6}\right)$ into these formulae instead of $A_{\nu 6}$.

We interpret the formal limit II as the asymptotics as $n \rightarrow+\infty$ of the sequence $A_{\nu 6}^{2 n}\left(\varepsilon_{n} t_{5}\right)$, or, equivalently, as the asymptotics of the corresponding sequences of their matrix elements. Here $t_{5}$ is a real positive number, which is further assumed as a given parameter. Asymptotics of the odd sequence, $A_{\nu 6}^{2 n+1}\left(\varepsilon_{n} t_{5}\right)$, is given by exactly the same formulae as for the even one, $A_{\nu 6}^{2 n}\left(\varepsilon_{n} t_{5}\right)$, (see Equations (4.11)-4.17) with $\varepsilon \rightarrow \varepsilon_{n}$ ) but in the corresponding formulae for the monodromy data one has to change $\Theta_{6} \rightarrow \Theta_{6}-1$. In the derivation of the results stated in Theorem 6.1 below, we omit the super/subscript $n$, if it does not cause a confusion.

To formulate our result we need to introduce some preliminary notation. It follows from Equations (3.7), (6.1), and (6.2) that $e^{ \pm \pi i \Theta_{6}}$ are the eigenvalues of $M_{t 6}$ and $e^{ \pm \pi i\left(\Theta_{\infty 5}-\Theta_{6}\right)}$ are the eigenvalues of $M_{06}$. Define

$$
\begin{gathered}
\mathbf{T}=\operatorname{tr}\left(M_{t 6}-e^{+\pi i \Theta_{6}}\right)\left(M_{06}-e^{-\pi i\left(\Theta_{\infty 5}-\Theta_{6}\right)}\right), \\
l=\frac{1}{\pi i} \ln \left(\cos \left(\pi \Theta_{\infty 5}\right)-\frac{1}{2} \mathbf{T} \pm \sqrt{\left(\cos \left(\pi \Theta_{\infty 5}\right)-\frac{1}{2} \mathbf{T}\right)^{2}-1}\right), \quad-1<\operatorname{Re} l<1 \\
\alpha=-\frac{1}{2}\left(\Theta_{\infty 5}-l\right), \quad \beta=-\frac{1}{2}\left(\Theta_{\infty 5}+l\right) .
\end{gathered}
$$

The sign before the square root in Equation (6.5) can be chosen arbitrary: the change of the sign means simply the change $\alpha \leftrightarrow \beta$ in our construction, which is invariant under this transformation.

Theorem 6.1 Let $\mu_{6} \in \mathcal{M}_{6}\left(\Theta_{\infty 5}-\Theta_{6}, \Theta_{16}, \Theta_{6}, \Theta_{\infty 6}\right)$. Suppose the following conditions are valid:

1. (6.1), (6.2), and $\Theta_{\nu 6} \in \mathbb{C} \backslash \mathbb{Z}, \nu=0,1, \infty$;

2. $\alpha, \beta, l \in \mathbb{C} \backslash \mathbb{Z}$;

3. $\left(M_{t 6}-e^{-\pi i \Theta_{6}}\right)\left(M_{06}-e^{-\pi i\left(\Theta_{\infty 5}-\Theta_{6}\right)}\right) \neq 0$.

4. The inverse monodromy problem for Equation (3.1) is solvable for all pairs $\left(\mu_{6}, t_{6}\right)$ such that $t_{6}=\varepsilon_{n} t_{5}>0$, where $\varepsilon_{n}$ is given by Equation (6.1).

5. It is possible to define the sequence $A_{\nu 6}^{2 n}\left(\varepsilon_{n} t_{5}\right)$ : it is true in particular, if $\mu_{6}$ corresponds to the non-classical solution of Equation (3.16) ${ }^{8}$.

Then define $\mu_{5} \in \mathcal{M}_{5}\left(\Theta_{05}, \Theta_{15}, \Theta_{\infty 5}\right)$ :

$$
\begin{gathered}
\Theta_{05}=\Theta_{\infty 6}, \quad \Theta_{15}=\Theta_{16}, \\
S_{0}=\left(\begin{array}{cc}
1 & 0 \\
-\frac{2 \pi i}{\Gamma(1-\alpha) \Gamma(1-\beta)} & 1
\end{array}\right), \quad S_{1}=\left(\begin{array}{cc}
1 & -\frac{2 \pi i}{\Gamma(\alpha) \Gamma(\beta)} e^{\pi i \Theta_{\infty 5}} \\
0 & 1
\end{array}\right), \\
K M_{\infty 6} K^{-1}=M_{05}, \quad K M_{16} K^{-1}=M_{15},
\end{gathered}
$$

\footnotetext{
${ }^{8}$ The last condition is not necessary: such sequence is possible to organize for the classical solutions too, just for some of them $n \in \mathbb{Z}_{-}$instead of $\mathbb{Z}_{+}$; we do not discuss here the corresponding modifications.
} 
where $K$ is the unique (up to the sign) solution of the system:

$$
K M_{t 6} K^{-1}=S_{0} e^{\pi i \Theta_{6} \sigma_{3}}, \quad K M_{06} K^{-1}=e^{-\pi i \Theta_{6} \sigma_{3}} S_{1} e^{\pi i \Theta_{\infty 5} \sigma_{3}} .
$$

See Theorem A.1 in the Appendix and Equations (A.19)-A.21).

If for a given pair $\left(\mu_{5}, t_{5}\right)$ the inverse monodromy problem is solvable, then an asymptotic expansion of the GSD $A_{\nu 6}^{2 n}\left(\varepsilon_{n} t_{5}\right)$ as $\varepsilon_{n} \rightarrow+0$ is given by Equations (4.21)-(4.24) with $\varepsilon=\varepsilon_{n}$.

Derivation. Suppose that the matrices $A_{\nu 6}$ in Equation (3.1) satisfy Equations (4.21)(4.24) corresponding to the formal limit II. Then one proves that in the domain $\lambda_{6} \in$ $\Omega_{\text {out }}:=\left\{\lambda_{6} \in \mathbb{C}, \varepsilon /\left|\lambda_{6}\right|^{3} \leq \mathcal{O}\left(\varepsilon^{3 \delta_{0}-2}\right),\left|\arg \lambda_{6}+\frac{\pi}{2}\right|<\delta_{1}, \delta_{0}>\frac{2}{3}, 0 \leq \delta_{1}<\pi\right\}$ the $\Psi_{6}$ function has the following asymptotics

$$
\Psi_{6}\left(\lambda_{6}, t_{6}\right) K^{-1}=\varepsilon^{\sigma_{3}} R \Psi_{5}^{0}\left(\lambda_{5}, t_{5}\right)(I+o(1)),
$$

where $K, R \in \mathrm{SL}(2, \mathbb{C}), R=\varepsilon^{-\sigma_{3}} R_{t 6}+o(1), \varepsilon^{-\sigma_{3}} R_{t 6}=\mathcal{O}(1)$. The matrix $K$ in Equation (6.11) is independent of $t_{\nu}, \lambda_{\nu}, \nu=5,6$ and $\varepsilon$. Equations (6.9) are an immediate consequence of (6.11) and (4.21). To this end our problem is to find $K$. To solve it we consider the function $\Phi$ which solves the hypergeometric equation:

$$
\frac{d \Phi}{d \lambda_{6}}=\left(\frac{A_{06}}{\lambda_{6}}+\frac{A_{16}}{\lambda_{6}-t_{6}}\right) \Phi,
$$

and has the same monodromy matrices at regular singularities $\lambda_{6}=0$ and $t$ as the function $\Psi_{6}\left(\lambda_{6}, t_{6}\right) K^{-1}$. We call the latter property of $\Phi$ as the condition $M$. The fundamental solution of Equation (6.12) can be presented as

$$
\Phi=P Y(x), \quad x=\lambda_{6} / t_{6},
$$

where $Y(x)$ is given by (5.20) with the parameters $\alpha, \beta, \gamma$ satisfying the equations

$$
1-\gamma=\Theta_{06}, \quad \gamma-1-\alpha-\beta=\Theta_{t 6}, 0<|\beta-\alpha| \mid<1,
$$

and $P^{-1} A_{\nu 6} P \approx A_{\frac{\nu}{t} Y}$, where $A_{\frac{\nu}{t} Y}$ is defined by (5.27) and (5.28). The last condition in (6.14) is assumed by taking into account the theorem formulated in [1] [ $\S 2$, pp.1145-1146]. Now we have to determine $l=\alpha-\beta$. To do this we use condition $M$ in the following way. First, for $m=0,1$ we calculate the matrix $\hat{K}(2 m-1)$ by help of the equation

$$
\Phi \hat{K}^{-1}(2 m-1)=\varepsilon^{\sigma_{3}} R \Psi_{5}^{m}, \quad \lambda_{6} \sim \mathcal{O}\left(\varepsilon^{1-\delta_{0}}\right) .
$$

Thus, using definitions of Section 3 we find the Stokes multiplier

$$
S_{0}=\hat{K}(-1) \hat{K}^{-1}(1),
$$

and the monodromy matrix at the infinity point

$$
M_{\infty 5}=\hat{K}(-1) M_{1 Y} M_{0 Y} \hat{K}^{-1}(-1) .
$$

Now the direct calculation shows

$$
\hat{K}(-1) M_{1 Y} \hat{K}^{-1}(-1)=S_{0} e^{\pi i \Theta_{t 6} \sigma_{3}} .
$$


Comparing Equation (3.48) for $k=0$ with Equations (6.17) and (6.18) we obtain the other Stokes multiplier:

$$
\hat{K}(-1) M_{0 Y} \hat{K}^{-1}(-1)=e^{\pi i \Theta_{06} \sigma_{3}} e^{-\pi i \Theta_{\infty 5} \sigma_{3}} S_{1} e^{\pi i \Theta_{\infty 5} \sigma_{3}} .
$$

Equations (6.16) and (6.19) are equivalent to the ones presented in (6.8), but the values of the parameters $\alpha$ and $\beta$ still remain undetermined. Comparing Equations (6.15), (6.13), and (6.11), one finds

$$
K M_{\nu 6} K^{-1}=\hat{K}(-1) M_{\frac{\nu}{t} Y} \hat{K}^{-1}(-1), \quad \nu=0, t .
$$

Now Equations (6.20), 66.19), and (6.18) yield (6.10), which are the exact formulae, since both sides of these equations are independent of $\varepsilon$. Finally we use Theorem A.1 of the Appendix to find formulae (6.4) - 6.6) and the matrix $K$.

The rigorous aspect of the above calculation is based on the justification scheme suggested in [35]. The scheme can be explained as follows. Suppose that the inverse monodromy problem for Equation (3.40) is solvable for a given value of the parameter $t_{5}$ in some neighborhood $\mathcal{O}\left(\mu_{5}\right)$ of the given point $\mu_{5} \in \mathcal{M}_{5}\left(\Theta_{05}, \Theta_{15}, \Theta_{\infty 5}\right)$. Thus for all $\tilde{\mu}_{5} \in \mathcal{O}\left(\mu_{5}\right)$ we have functions $\tilde{u}_{5}, \tilde{z}_{5}$, and $\tilde{y}_{5}$ whose monodromy data coincide with $\tilde{\mu}_{5}$. Then using Equations (4.26) - 4.32), and (4.21) with $\tilde{u}_{5}, \tilde{z}_{5}$, and $\tilde{y}_{5}$ instead of $u_{5}, z_{5}$, and $y_{5}$ and the discrete $\varepsilon=\varepsilon_{n}$, substitute them into the matrix elements of the residue matrices of Equation (3.1). Then the derivation presented above can be interpreted as as the proof that the monodromy data $\hat{\mu}_{5}$, of thus obtained Equation (3.1), differ from the data $\tilde{\mu}_{5}$, obtained by the inversion of Equations (6.9) and (6.10), on quantities estimated as $\|\tilde{\mu}-\hat{\mu}\|<o(1)$. To be able to apply the scheme of justification suggested in [35] the last estimate must possess an important property of "local uniformness": this means that there exists a neighborhood of $\tilde{\mu}_{5}$ such that the above estimate is uniform for all points $\hat{\mu}_{5}$ in this neighborhood. In the terminology of the work [35: the inverse monodromy problem for Equation (3.1) for the monodromy data $\tilde{\mu}_{5}$ is asymptotically solvable. The main result of [35] says that if the monodromy problem is asymptotically solvable, then it is exactly solvable. Since the solution of the inverse monodromy problem is unique, this solution coincides with the GSD introduced in the beginning of this section.

Now I am going to give some details to the calculation outlined above. Let us begin with the matching (6.15) as it is the most crucial point. Consider Equation (6.13). The matrix $P$ which maps the function $Y(x)$ into a fundamental solution of the hypergeometric equation (6.12) is defined as follows

$$
P^{-1} A_{\nu 6} P=A_{\frac{\nu}{t} Y}+\mathcal{O}\left(\varepsilon^{\delta_{0}-1}\right), \quad \nu=0,1, \quad P \in \mathrm{SL}(2, \mathbb{C}) .
$$

Let's explain the estimate $\mathcal{O}\left(\varepsilon^{\delta_{0}-1}\right)$ in Equation (6.21): it is an important and rather subtle moment. First we show that the estimate cannot be less than $\mathcal{O}(1)$. Actually, summing up equations in (6.21) (with the error estimate $\mathcal{O}(1)$ ) and using the second equality in (5.26) and the first equation in (3.2), one arrives at

$$
P^{-1}\left(\frac{\Theta_{\infty 6}}{2} \sigma_{3}+A_{16}\right) P=-\frac{\beta-\alpha}{2} \sigma_{3}+\mathcal{O}(1) .
$$

Suppose that in Equation (6.22) one have a better estimate, $o(1)$, instead of $\mathcal{O}(1)$. Then it implies

$$
\operatorname{det}\left(\frac{\Theta_{\infty 6}}{2} \sigma_{3}+A_{16} \pm \frac{\beta-\alpha}{2}\right)=o(1) .
$$


The last equation leads to the nontrivial dependence of $\alpha$ and $\beta$ from $t_{5}$ (see Equations (4.27) and the first equation (3.12) with $\nu=1$. This dependence contradicts the isomonodromy condition, since the monodromy data in particular Stokes multipliers $S_{k}$ must be independent of $t_{5}$ (see (6.8)). Thus the minimal possible error estimate in Equation (6.21) is $\mathcal{O}(1)$, i.e., $\delta_{0} \leq 1$. In the last case Equation (6.22) becomes uninformative.

One of the most important moments of our derivation is the matching procedure of $\Phi$ with $\varepsilon^{\sigma_{3}} R_{t 6} \Psi_{5}^{0}$. This matching occurs at the domain $\lambda_{6} \sim \varepsilon^{1-\delta_{0}}, \frac{2}{3}<\delta_{0}<1$, where the lower limit for $\delta_{0}$ is defined via the matching of $\Psi_{6}$ and $R_{t 6} \Psi_{5}^{0}$ (see Equation (6.11)): The term $t_{6}^{2} A_{t 6} / \lambda_{6}^{3} \sim \mathcal{O}\left(\varepsilon^{3 \delta_{0}-2}\right)$, which was neglected in Equation (13.1) to obtain the equation for $R_{t 6} \Psi_{5}^{0}$, should decrease. The upper limit for $\delta_{0}: \delta_{0}<1$ is originated from the matching of $\Psi_{6}$ and $\Phi$ : the corresponding equations differ by the term $\mathcal{O}\left(t_{6} A_{t 6} / \lambda_{6}^{2}\right) \sim \mathcal{O}\left(\varepsilon^{2\left(\delta_{0}-1\right)}\right)$. Thus to guarantee the matching we must define $P$ in Equation (6.21) with the error estimate not worse than $\lambda_{6} \mathcal{O}\left(\varepsilon^{2\left(\delta_{0}-1\right)}\right)=\mathcal{O}\left(\varepsilon^{\delta_{0}-1}\right)$. To summarize: the primary qualities of $\Phi$ (according to [35]) are its monodromy data and the matching with $\varepsilon^{\sigma_{3}} R_{t 6} \Psi_{5}^{0}$. It is because of these properties the function $\Phi$, in fact, is not the exact solution of Equation (6.12), but solves it up to the leading order with the rather large error $\mathcal{O}\left(\varepsilon^{\delta_{0}-1}\right)$. It seems that the function $\Phi$ with the required properties can be defined to satisfy Equation (6.12) more precisely, with the error $\mathcal{O}(1)$, but for this goal we need to substantially increase complexity of our calculations. In any case we cannot define the function $\Phi$ to satisfy Equation (6.12) with the error less than $\mathcal{O}(1)$, because it contradicts the matching procedure. Actually, the asymptotic expansion for $A_{16}$, which we substitute into Equation (6.23) to get the contradiction, is obtained from the differential equation for $R_{t 6} \Psi_{5}^{0}$ and, hence, it is inexplicit consequence of the matching.

Taking into account the discussion above, we rewrite System (6.21) with the error $\mathcal{O}\left(\varepsilon^{\delta_{0}-1}\right)$ changed by $\mathcal{O}(1)$ as follows:

$$
\begin{aligned}
(-1)^{\frac{\nu}{t}} \frac{\beta(1-\gamma)}{\beta-\alpha} & =\left(d-\frac{b \varepsilon^{2}}{u_{\nu 6}}\right)\left(b \Theta_{\nu 6}-\frac{u_{\nu 6} z_{\nu 6}}{\varepsilon^{2}}\left(d-\frac{b \varepsilon^{2}}{u_{\nu 6}}\right)\right)+\mathcal{O}(1) \\
(-1)^{1+\frac{\nu}{t}} \frac{\alpha(1-\gamma)}{\beta-\alpha} & =\left(c-\frac{a \varepsilon^{2}}{u_{\nu 6}}\right)\left(\frac{u_{\nu 6} z_{\nu 6}}{\varepsilon^{2}}\left(c-\frac{a \varepsilon^{2}}{u_{\nu 6}}\right)-a \Theta_{\nu 6}\right)+\mathcal{O}(1) \\
(-1)^{\frac{\nu}{t}} \frac{(\alpha+\beta)(1-\gamma)}{2(\beta-\alpha)} & =\left(d-\frac{b \varepsilon^{2}}{u_{\nu 6}}\right)\left(c-\frac{a \varepsilon^{2}}{u_{\nu 6}}\right) \frac{u_{\nu 6} z_{\nu 6}}{\varepsilon^{2}} \\
& -\frac{\Theta_{\nu 6}}{2}\left(a\left(d-\frac{b \varepsilon^{2}}{u_{\nu 6}}\right)+b\left(c-\frac{a \varepsilon^{2}}{u_{\nu 6}}\right)\right)+\mathcal{O}(1)
\end{aligned}
$$

where $\nu=0, t$ and $a, b, c$, and $d$ are the matrix elements of $P$ :

$$
\begin{gathered}
P=\varepsilon^{\sigma_{3}}\left(\begin{array}{ll}
a & b \\
c & d
\end{array}\right), \quad \text { with } a, b, c, d=\mathcal{O}(1), \\
a d-b c=1 .
\end{gathered}
$$

Using now Formulae (4.26), (4.28), 6.14), and (6.25), one rewrites Equations (6.24) as follows:

$$
u_{\nu 6}=\varepsilon^{2} \frac{a+b}{c+d}+\mathcal{O}\left(\varepsilon^{3}\right), \quad \frac{z_{\nu 6}}{\Theta_{\nu 6}}=\frac{(a+b)(c+d)}{2}\left(\frac{b-a}{b+a}-\frac{\beta-\alpha}{\beta+\alpha}\right)+\mathcal{O}(\varepsilon), \quad \nu=0, t .
$$


To determine the matrix $P$ uniquely we must add to (6.26) and (6.27) one more equation. We get it via the matching (6.15): the matching domain now is

$$
x=\frac{\lambda_{6}}{t_{6}}=\left(\varepsilon \lambda_{5} t_{5}\right)^{-1}=\mathcal{O}\left(\varepsilon^{-\delta_{0}}\right), \quad \frac{2}{3}<\delta_{0}<1,
$$

so that Asymptotics (5.29), (5.31) imply the following equations:

$$
\begin{gathered}
\varkappa=2 m-1, \quad \text { for } m=0,1 \quad \alpha+\beta=-\Theta_{\infty 5}, \\
\frac{\varepsilon^{\sigma_{3}}}{\sqrt{\alpha-\beta}}\left(\begin{array}{ll}
a & b \\
c & d
\end{array}\right)\left(\begin{array}{cc}
1 & \beta \\
1 & \alpha
\end{array}\right) t^{-\frac{\Theta_{\infty 5}}{2} \sigma_{3}}=R_{t 6}+\varepsilon^{\sigma_{3}} \mathcal{O}\left(\varepsilon^{\tilde{\delta}}\right) .
\end{gathered}
$$

Recall the definition of $\mathcal{O}\left(\varepsilon^{\tilde{\delta}}\right)$ below Equation (5.31). The first equation in (6.2.) and (6.15) show that (6.16) and (6.17) hold up to the error bound $\mathcal{O}\left(\varepsilon^{\tilde{\delta}}\right)$. From (6.30) one finds that Equations (6.26) and (6.27) are valid up to the estimate $\mathcal{O}\left(\varepsilon^{\tilde{\delta}}\right)$. Equation (6.30) together with the first equation in (4.28) and the equality $\varepsilon_{n} \Theta_{t 6}=-1$ we arrive at:

$$
\begin{gathered}
a=-\frac{1}{s_{t 6} \sqrt{\alpha-\beta}}\left(\alpha t_{5}^{\frac{\Theta_{\infty 5}}{2}}+u_{5} t_{5}^{-\frac{\Theta_{\infty 5}}{2}}\right), \quad b=\frac{1}{s_{t 6} \sqrt{\alpha-\beta}}\left(\beta t_{5}^{\frac{\Theta_{\infty 5}}{2}}+u_{5} t_{5}^{-\frac{\Theta_{\infty 5}}{2}}\right), \\
c=\frac{s_{t 6}}{\sqrt{\alpha-\beta}}\left(\frac{\alpha}{u_{5}} \frac{z_{5}}{\Theta_{05}} t_{5}^{\frac{\Theta_{\infty 5}}{2}}+\left(\frac{z_{5}}{\Theta_{05}}+1\right) t_{5}^{-\frac{\Theta_{\infty 5}}{2}}\right), \\
d=-\frac{s_{t 6}}{\sqrt{\alpha-\beta}}\left(\frac{\beta}{u_{5}} \frac{z_{5}}{\Theta_{05}} t_{5}^{\frac{\Theta_{\infty 5}}{2}}+\left(\frac{z_{5}}{\Theta_{05}}+1\right) t_{5}^{-\frac{\Theta_{\infty 5}}{2}}\right) .
\end{gathered}
$$

Now one uses (6.16) and (6.29) to find, again up to $\mathcal{O}\left(\varepsilon^{\tilde{\delta}}\right)$, the first equation in (6.8). To finish the proof we have to confirm the second equation in (6.8). To do it, we have to prove (6.18) and to find $S_{1}$ from (6.19). Details for these calculations are as follows: apply the formulae for the $\Gamma$-function (5.41) and (5.42) to Equations (5.24) and (5.25) to find:

$$
\begin{gathered}
C_{\alpha \beta \gamma}^{0} \hat{K}^{-1}(-1)=D_{0}\left(1+\mathcal{O}\left(\varepsilon^{\tilde{\delta}}\right)\right)\left(\begin{array}{cc}
1 & -\frac{\pi \exp (\pi(\alpha+\beta+1-\gamma))}{(\alpha-\beta) \sin (\pi \gamma) \Gamma(\alpha) \Gamma(\beta)} \\
0 & 1
\end{array}\right) \frac{1}{\sqrt{\alpha-\beta}}\left(1+\mathcal{O}\left(\varepsilon^{\sigma_{3}}\right)\right) \\
C_{\alpha \beta \gamma}^{1} \hat{K}^{-1}(-1)=D_{1}\left(1+\mathcal{O}\left(\varepsilon^{\tilde{\delta}}\right)\right)\left(\begin{array}{cc}
1 & 0 \\
\frac{\pi \exp (\pi(\gamma-\alpha-\beta))(\beta-\alpha)}{\sin (\pi(\alpha+\beta-\gamma)) \Gamma(1-\alpha) \Gamma(1-\beta)} & 1
\end{array}\right)\left(1+\mathcal{O}\left(\varepsilon^{\tilde{\delta}}\right)\right)
\end{gathered}
$$

where $D_{k}$ are diagonal matrices. Finally, one uses the definition of $M_{\nu Y}$ :

$$
M_{\nu Y}=\left(C_{\alpha \beta \gamma}^{\nu}\right)^{-1} e^{\pi i \Theta_{t \cdot \nu 6} \sigma_{3}} C_{\alpha \beta \gamma}^{\nu}, \quad \nu=0,1
$$

to prove (6.18) and (6.19).

The result of Theorem 6.1 can be immediately generalized as follows. For $k=1, \ldots, n$ define $a_{k} \in \mathbb{C} \backslash\{0\}, a_{k} \neq a_{l}$, for $k \neq l, \frac{\partial a_{k}}{\partial t_{6}}=\frac{\partial a_{k}}{\partial \lambda_{6}}=0$. Consider the functions $\Psi_{\nu}=$ $\Psi_{\nu}\left(\lambda_{\nu}, t_{\nu} ; a_{1}, \ldots, a_{n}\right) \in \mathrm{SL}(2, \mathbb{C}) \nu=5,6$, which generalize the functions $\Psi_{\nu}$ defined in Section 3. as the normalized (3.6) and (3.43) fundamental solutions of the systems:

$$
\begin{aligned}
\frac{d}{d \lambda_{6}} \Psi_{6} & =\left(\frac{A_{06}}{\lambda_{6}}+\frac{A_{t 6}}{\lambda_{6}-t_{6}}+\sum_{k=1}^{m} \frac{A_{k 6}}{\lambda_{6}-a_{k}}\right) \Psi_{6}, \\
\frac{d}{d t_{6}} \Psi_{6} & =-\frac{A_{t 6}}{\lambda_{6}-t_{6}} \Psi_{6},
\end{aligned}
$$




$$
\begin{gathered}
\frac{d}{d \lambda_{5}} \Psi_{5}=\left(\frac{t_{5}}{2} \sigma_{3}+\frac{A_{05}}{\lambda_{5}}+\sum_{k=1}^{m} \frac{A_{k 5}}{\lambda_{5}-1 / a_{k}}\right) \Psi_{5}, \\
\frac{d}{d t_{5}} \Psi_{5}=\left(\frac{\lambda_{5}}{2} \sigma_{3}+\frac{1}{t_{5}}\left(\frac{\Theta_{\infty 5}}{2} \sigma_{3}+\sum_{k=0}^{m} A_{k 5}\right)\right) \Psi_{5}, \\
A_{t 6}+\sum_{k=0}^{m} A_{k 6} \equiv-\frac{\Theta_{\infty 6}}{2} \sigma_{3}, \quad \operatorname{diag} \sum_{k=0}^{m} A_{k 5} \equiv-\frac{\Theta_{\infty 5}}{2} \sigma_{3}, \quad A_{k \nu} \in \operatorname{sl}_{2}(\mathbb{C}) .
\end{gathered}
$$

The local objects like $\Theta_{k \nu}, R_{k \nu}$, and $M_{k \nu}, k=1, \ldots, m$, are defined in the same manner as that in Section 3 for $k=1$ and $a_{1}=1$. Systems of isomonodromy deformations are the compatibility conditions for the systems of the linear ODEs written above, and the GSDs $A_{k 6}^{2 n}\left(\varepsilon_{n} t_{5}\right)$ can be defined as in the beginning of this section.

Corollary 6.1 Let conditions of Theorem 6.1 be valid for

$$
\mathcal{M}_{6}=\mathcal{M}_{6}\left(\Theta_{06}, \Theta_{t 6}, \Theta_{16}, \ldots, \Theta_{m 6}, \Theta_{\infty 6}\right) \text {, }
$$

then asymptotics $(n \rightarrow+\infty)$ of the GSDs $A_{k 6}^{2 n}\left(\varepsilon_{n} t_{5}\right),(k=1, \ldots, m)$, are given by Equations (4.21) - 4.23) which are supplemented by

$$
R_{t 6}^{-1} A_{k 6} R_{t 6}=A_{k 5}+\mathcal{O}(\varepsilon)
$$

Equation (4.24) should be generalized as follows

$$
-R_{t 6}^{-1} \frac{d}{d t_{5}} R_{t 6}=\frac{1}{t_{5}}\left(\frac{\Theta_{\infty 5}}{2} \sigma_{3}+\sum_{k=0}^{m} A_{k 5}\right)+\mathcal{O}(\varepsilon),
$$

and with the change $\varepsilon \rightarrow \varepsilon_{n}$ in all formulae. The monodromy data for the limiting $\Psi_{5}$ function is given by exactly the same formulae as that in Theorem 6.1 supplemented with the equations:

$$
\Theta_{k 6}=\Theta_{k 5}, \quad K M_{k 6} K^{-1}=M_{k 5}, \quad k=1, \ldots, m .
$$

Proposition 6.1 For the sixth Painlevé function (and the corresponding $\tau$-function) both limits considered in Section 4 are equivalent under the definition given in the Introduction.

Proof. Let us supply with the superscripts $I$ and $I I$ the objects corresponding to the first/second limits. Then for $\Psi_{6}$ function one finds:

$$
\Psi_{6}^{I I}\left(\lambda_{6}^{I I}, t_{6}\right)=t_{6}^{-\frac{\Theta_{05}}{2} \sigma_{3}}\left(R_{06}^{I}\right)^{-1} \Psi_{6}^{I}\left(\lambda_{6}^{I}, t_{6}\right)\left(C_{06}^{I}\right)^{-1}, \quad \lambda_{6}^{I I}=t_{6} / \lambda_{6}^{I}
$$

This transformation is controlling the limiting procedure. Thus it is not necessary to consider especially what is happening on the lower $\mathcal{M}$-plane of the diagram on Figure 3. Formula (6.34) generates the following transformation for the matrices $A_{\nu 6}$ :

$$
A_{\nu 6}^{I I}=t_{6}^{-\frac{\Theta_{06}^{I}}{2} \sigma_{3}}\left(R_{06}^{I}\right)^{-1} A_{\frac{t}{\nu} 6}^{I} R_{06}^{I} t_{6}^{\frac{\Theta_{06}^{I}}{2} \sigma_{3}}, \quad \nu=1, t
$$


For the formal monodromy one finds:

$$
\begin{gathered}
\Theta_{05}^{I I}=\Theta_{\infty 6}^{I I}=\Theta_{06}^{I}=\Theta_{05}^{I}, \\
\Theta_{15}^{I I}=\Theta_{16}^{I I}=\Theta_{t 6}^{I}=-\Theta_{15}^{I}, \\
\Theta_{t 6}^{I I}=\Theta_{16}^{I}=-\frac{1}{\varepsilon}, \\
\Theta_{06}^{I I}=\Theta_{\infty 6}^{I} \quad \Theta_{\infty 6}^{I}+\Theta_{16}^{I}=\Theta_{06}^{I I}+\Theta_{t 6}^{I I}=\Theta_{\infty 5} .
\end{gathered}
$$

Substituting (4.2) and (4.22) into Equation (6.34) one obtains

$$
R_{t 6}^{I I} \Psi_{5}^{I}\left(\lambda_{5}, t_{5}\right) K^{I I}=t_{6}^{-\frac{\Theta_{06}^{I}}{2} \sigma_{3}}\left(R_{06}^{I}\right)^{-1} R_{16}^{I} \Psi_{5}^{I}\left(\lambda_{5}, t_{5}\right) K^{I}\left(C_{06}^{I}\right)^{-1} .
$$

Where $K^{p}$ is the matrix introduced in Theorem $p(p=I, I I)$. Thus we have

$$
\begin{aligned}
R_{t 6}^{I I} f_{0}^{-\sigma_{3}} & =t^{-\frac{\Theta_{06}^{I}}{2} \sigma_{3}}\left(R_{06}^{I}\right)^{-1} R_{16}^{I}, \\
K^{I I} & =f_{0}^{-\sigma_{3}} K^{I}\left(C_{06}^{I}\right)^{-1} .
\end{aligned}
$$

Using Relations (6.36) and (6.37) one proves that Equations (4.3) and (4.23) are equiva-

lent. For the matrix elements one has to take into account that $\Theta_{15}^{I I}=-\Theta_{15}^{I}$ and, hence, Equations (3.52) and (3.53) yield

$$
u_{5}^{I I}=u_{5}^{I}, \quad z_{5}^{I I}=z_{5}^{I}, \quad y_{5}^{I I}\left(z_{5}+\frac{\Theta_{05}-\Theta 15^{I I}+\Theta_{\infty 5}}{2}\right)=y_{5}^{I}\left(z_{5}+\frac{\Theta_{05}-\Theta 15^{I}+\Theta_{\infty 5}}{2}\right) .
$$

The so-called c-problem discussed in the beginning of this Section reveals itself in Equations (6.36) and (6.37) as the ambiguity in the definitions of $R_{06}, C_{06}$ :

$$
R_{06} \rightarrow R_{06} c^{\sigma_{3}}, \quad C_{06} \rightarrow c^{-\sigma_{3}} C_{06} .
$$

\section{A Appendix}

For $\nu=0,1$ consider the matrices

$$
M_{\nu 6}=\left(\begin{array}{ll}
a_{\nu} & b_{\nu} \\
c_{\nu} & d_{\nu}
\end{array}\right) \in \mathrm{SL}(2, \mathbb{C})
$$

with the eigenvalues $r_{\nu} \in \mathbb{C} \backslash\{0, \pm 1\}$. The numbers $1 / r_{\nu}$ have the same properties. For the matrix elements one finds

$$
b_{\nu} c_{\nu}=-\left(a_{\nu}-r_{\nu}\right)\left(a_{\nu}-1 / r_{\nu}\right) .
$$

Define the upper- and lower-triangular matrices:

$$
M_{0 \Delta}=\left(\begin{array}{cc}
r_{0} & f_{0} \\
0 & 1 / r_{0}
\end{array}\right), \quad M_{1 \Delta}=\left(\begin{array}{cc}
r_{1} & 0 \\
f_{1} & 1 / r_{1}
\end{array}\right),
$$

where $f_{\nu} \in \mathbb{C}$. Here we study the following problem.

Problem: For given matrices $M_{\nu 6}$ find the parameters $f_{\nu}$ such that there exists the matrix $K \in \mathrm{SL}(2, \mathbb{C})$ which solves

$$
K M_{\nu 6}=M_{\nu \Delta} K, \quad \nu=0,1 .
$$

The solution of the problem is formulated as Theorem A.1 below. 
Proposition A.1 The problem is unsolvable if the matrices $M_{\nu 6}$ satisfy one of the following equations:

$$
\begin{gathered}
\left(M_{06}-r_{0}\right)\left(M_{16}-r_{1}\right)=0, \\
\left(M_{16}-1 / r_{1}\right)\left(M_{06}-1 / r_{0}\right)=0 .
\end{gathered}
$$

Proof. Suppose the problem is solvable and one of the equations, say, A.3 holds. Then there exists the matrix $K$ satisfying Equation (A.2). Using it one finds;

$$
\begin{aligned}
0=K 0 K^{-1}= & K\left(M_{06}-r_{0}\right)\left(M_{16}-r_{1}\right) K^{-1}=\left(M_{0 \Delta}-r_{0}\right)\left(M_{1 \Delta}-r_{1}\right) \\
= & \left(\begin{array}{cc}
0 & f_{0} \\
0 & 1 / r_{0}-r_{0}
\end{array}\right)\left(\begin{array}{cc}
0 & 0 \\
f_{1} & 1 / r_{1}-r_{1}
\end{array}\right) \\
= & \left(\begin{array}{cc}
f_{0} f_{1} & -f_{0}\left(r_{1}-1 / r_{1}\right) \\
-f_{1}\left(r_{0}-1 / r_{0}\right) & \left(r_{0}-1 / r_{0}\right)\left(r_{1}-1 / r_{1}\right)
\end{array}\right) .
\end{aligned}
$$

This equation contradicts to the condition $r_{\nu}-1 / r_{\nu} \neq 0$. One arrives at the same contradiction by making analogous calculations for Equation (A.4).

Remark A.1 Equations (A.3) and A.4 are equivalent. To prove it define the eigenvectors, $e_{\nu \epsilon}, \nu=0,1, \epsilon=-1,1$ :

$$
\left(M_{\nu 6}-r_{\nu}^{\epsilon}\right) e_{\nu \epsilon}=0, \quad\left\|e_{\nu \epsilon}\right\|_{\mathbb{C}^{2}}=1 .
$$

Each pair $\left(e_{01}, e_{0-1}\right)$ and $\left(e_{11}, e_{1-1}\right)$ is a basis in $\mathbb{C}^{2}$. If Equation (A.3) holds, then we can choose $e_{01}=e_{1-1}$. Thus, if $x \in \mathbb{C}^{2}$, then $x=\alpha e_{01}+\beta e_{0-1}$, where $\alpha, \beta \in \mathbb{C}$, and

$$
\left(M_{16}-1 / r_{1}\right)\left(M_{06}-1 / r_{0}\right) x=\alpha\left(M_{16}-1 / r_{1}\right) e_{01}=\alpha\left(M_{16}-1 / r_{1}\right) e_{1-1}=0 .
$$

Similarly vice versa, from (A.4) to (A.3).

Proposition A.2 The problem is solvable with $f_{0}=f_{1}=0$ iff the matrices $M_{\nu 6}$ are commuting,

$$
M_{06} M_{16}=M_{16} M_{06},
$$

and don't satisfy Equations (A.3) and (A.3). The general solution of Equation (A.2) can be presented as

$$
K=k^{\sigma_{3}} K_{0}, \quad k \in \mathbb{C} \backslash\{0\}, \quad K_{0}^{-1}=\left(e_{01}, e_{0-1}\right)
$$

where $e_{0 \epsilon}$ are the column eigenvectors of $M_{06}$ defined in Remark A.1.

Proof. Multiplying 1.-h.s. of Equation (A.8) by $K_{0}$ and the r.-h.s. by $K_{0}^{-1}$ and taking into account that $K_{0} M_{06} K^{-1}=r_{0}^{\sigma_{3}}$ one finds that $K_{0} M_{16} K^{-1}$ is a diagonal matrix and, hence, $K_{0} M_{16} K^{-1}=r_{1}^{\delta \sigma_{3}}$, where $\delta= \pm 1$. Thus, if $\delta=+1$, then $k^{\sigma_{3}} K_{0}$ is a solution of Equations (A.2) with $f_{0}=f_{1}=0$; if $\delta=-1$, then the matrices $M_{\nu 6}$ satisfy Equations (A.3) and A.4 and the problem is unsolvable.

Let $L$ be a matrix that solves Equations (A.2) for some $f_{0}$ and $f_{1}$. Then multiplying both parts of Equation (A.8) by $L$ from 1.-h.s. and by $L^{-1}$ from r.-h.s. and using Equations (A.2) one finds that $f_{0}=f_{1}=0$. Conversely, suppose that we can present $M_{\nu 6}$ as $L^{-1} r_{\nu}^{\sigma_{3}} L$ and hence the commutator $\left[M_{06}, M_{16}\right]$ vanishes and Equations (A.3) and (A.4) are not valid. 
Proposition A.3 If noncommuting matrices $M_{\nu 6}\left(\left[M_{06}, M_{16}\right] \neq 0\right)$ satisfy one of the following equations:

$$
\begin{aligned}
& \left(M_{06}-r_{0}\right)\left(M_{16}-1 / r_{1}\right)=0, \\
& \left(M_{16}-r_{1}\right)\left(M_{06}-1 / r_{0}\right)=0,
\end{aligned}
$$

then the problem is solvable. For all solutions:

$$
f_{1}=0, \quad f_{0} \in \mathbb{C} \backslash\{0\} .
$$

Conversely, if the problem is solvable with $f_{1}=0$, then Equations (A.9) and (A.10) are valid. For any pair $\left(f_{0}, f_{1}\right)$ satisfying (A.11) there are two solutions $K$ of System (A.2). These solutions differ only by a choice of the sign and can be written as follows:

$$
K^{-1}=\left(e_{11}, e_{1-1}\right) \varkappa_{1}^{\sigma_{3}}, \quad \varkappa_{1}=\sqrt{\frac{\alpha_{0}\left(r_{0}-1 / r_{0}\right)}{f_{0}}},
$$

where the column eigenvectors $e_{\nu \epsilon}, \nu=0,1, \epsilon=1,-1$, are defined as $\left(M_{\nu 6}-r_{\nu}^{\epsilon}\right) e_{\nu \epsilon}=0$, $\operatorname{det}\left(e_{\nu 1}, e_{\nu-1}\right)=1, e_{11}=e_{01}$, the last equation is always possible due to Equations (A.9) and (A.10), and $\alpha_{0}, \beta_{0} \in \mathbb{C} \backslash\{0\}$ are coefficients in the expansion $e_{1-1}=\alpha_{0} e_{01}+\beta_{0} e_{0-1}$.

Remark A.2 Equations (A.9) and (A.10) are equivalent. The proof can be done in the same manner as the one in Remark A.1 for the equivalence of (A.3) and (A.4).

Remark A.3 The eigenvalues $\left(e_{\nu 1}, e_{\nu-1}\right)$ are defined up to a parameter

$$
a_{\nu} \in \mathbb{C} \backslash\{0\}: \quad\left(e_{\nu 1}, e_{\nu-1}\right) \rightarrow\left(e_{\nu 1}, e_{\nu-1}\right) a_{\nu}^{\sigma_{3}} .
$$

Under this transformation $\varkappa_{\nu}$ is also changing as $\varkappa_{\nu} \rightarrow \varkappa_{\nu} / a_{\nu}$, so that $\varkappa_{1}$ in (A.12), by a proper choice of the basis, can be defined with $\alpha_{0}=f_{0}=1$ with the only ambiguity in the branch of the square root.

Proof. Consider the following equation:

$$
\begin{gathered}
K\left(M_{06}-r_{0}\right)\left(M_{16}-1 / r_{1}\right) K^{-1} \\
=\left(\begin{array}{cc}
0 & f_{0} \\
0 & 1 / r_{0}-r_{0}
\end{array}\right)\left(\begin{array}{cc}
r_{1}-1 / r_{1} & 0 \\
f_{1} & 0
\end{array}\right)=\left(\begin{array}{cc}
f_{0} f_{1} & 0 \\
f_{1}\left(1 / r_{0}-r_{0}\right) & 0
\end{array}\right) .
\end{gathered}
$$

It proves both: if the problem is solvable and $f_{1}=0$, then Equation (A.9) holds; and if Equation (A.9) is valid, then $f_{1}=0$.

Suppose (A.9) holds, define matrix $K$ by the first equation in A.12 with a parameter $\varkappa_{1} \in \mathbb{C} \backslash\{0\}$. This matrix $K$ solves Equation (A.2) with $\nu=1$ and $f_{1}=0$. Substituting thus defined matrix $K$ into Equation (A.2) with $\nu=0$ and $f_{0} \in \mathbb{C} \backslash\{0\}$ one proves that it becomes an identity iff $\varkappa_{1}$ is given by the second equation A.12. Note that $\left(f_{0}, f_{1}\right) \equiv(0,0)$ cannot be a solution of the problem since $\left[M_{06}, M_{16}\right] \neq 0$.

Proposition A.4 If noncommuting matrices $M_{\nu 6}\left(\left[M_{06}, M_{16}\right] \neq 0\right)$ satisfy one of the following equations:

$$
\begin{aligned}
& \left(M_{06}-1 / r_{0}\right)\left(M_{16}-r_{1}\right)=0 \\
& \left(M_{16}-1 / r_{1}\right)\left(M_{06}-r_{0}\right)=0
\end{aligned}
$$


then the problem is solvable. For all solutions:

$$
f_{0}=0, \quad f_{1} \in \mathbb{C} \backslash\{0\} .
$$

Conversely, if the problem is solvable with $f_{0}=0$, then Equations (A.13) and (A.14) are valid. For any pair $\left(f_{0}, f_{1}\right)$ satisfying (A.15) there are two solutions $K$ of System (A.2). These solutions differ only by a choice of the sign and can be written as follows:

$$
K^{-1}=\left(e_{01}, e_{0-1}\right) \varkappa_{0}^{\sigma_{3}}, \quad \varkappa_{0}=\sqrt{\frac{f_{1}}{\beta_{1}\left(1 / r_{1}-r_{1}\right)}},
$$

where the column eigenvectors $e_{\nu \epsilon}, \nu=0,1, \epsilon=1,-1$, are defined as $\left(M_{\nu 6}-r_{\nu}^{\epsilon}\right) e_{\nu \epsilon}=$ 0 , $\operatorname{det}\left(e_{\nu 1}, e_{\nu-1}\right)=1, e_{1-1}=e_{0-1}$, the last equation is always possible due to Equations (A.13) and (A.14), and $\alpha_{1}, \beta_{1} \in \mathbb{C} \backslash\{0\}$ are coefficients in the expansion $e_{01}=$ $\alpha_{1} e_{11}+\beta_{1} e_{1-1}$.

Remark A.4 Equations (A.13) and (A.14) are equivalent; the proof is analogous to the one in Remark A.1. As for the ambiguity in Equation (A.16), see Remark A.3

Proof. The proof is analogous to the one for Proposition A.3. The details are left to the interested reader.

Theorem A.1 The solution of the problem is described by the following cases:

1. If $\operatorname{tr}\left(M_{06}-1 / r_{0}\right)\left(M_{16}-r_{1}\right)=0$ and $\left[M_{06}, M_{16}\right] \neq 0$, then the problem is solvable. In this case either Equations (A.9), A.10) or (A.13), (A.14) are valid and the solution is is given in Proposition A.3 or A.4, respectively;

2. If $\operatorname{tr}\left(M_{06}-1 / r_{0}\right)\left(M_{16}-r_{1}\right)=0$ and $\left[M_{06}, M_{16}\right]=0$, then the problem is solvable. The solution is given in Proposition A.2.

3. If $\operatorname{tr}\left(M_{06}-1 / r_{0}\right)\left(M_{16}-1 / r_{1}\right)=0$, then there are two subcases:

3a. Equations (A.3) and (A.4) are valid. This is unsolvable case: apply Proposition A.1:

3b. $\left(M_{06}-1 / r_{0}\right)\left(M_{16}-1 / r_{1}\right)=0$. The problem is solvable. All solutions are the pairs $\left(f_{0}, f_{1}\right)$, such that $f_{0}, f_{1} \in \mathbb{C} \backslash\{0\}$ and

$$
f_{0} f_{1}=-\left(r_{0}-1 / r_{0}\right)\left(r_{1}-1 / r_{1}\right)
$$

For any pair $\left(f_{0}, f_{1}\right)$ satisfying Equation (A.17), there are two solutions, $K$ and $-K$, of System (A.2). Formula (A.19) for different choices of the branches of the square root in A.20 gives both, $K$ and $-K$; and

4. If Conditions 1-3 do not valid, then the problem is solvable: all solutions are the pairs $\left(f_{0}, f_{1}\right)$ such that $f_{0}, f_{1} \in \mathbb{C} \backslash\{0\}$ and

$$
\begin{aligned}
f_{0} f_{1} & =\operatorname{tr}\left(M_{1}-r_{1}\right)\left(M_{0}-1 / r_{0}\right)=\operatorname{tr}\left(M_{1}-1 / r_{1}\right)\left(M_{0}-r_{0}\right) \\
& =\operatorname{tr}\left(M_{0}-1 / r_{0}\right)\left(M_{1}-1 / r_{1}\right)-\left(r_{0}-1 / r_{0}\right)\left(r_{1}-1 / r_{1}\right) \\
& =\left(r_{0}+r_{1}\right)\left(1 / r_{0}+1 / r_{1}\right)-\operatorname{det}\left(M_{0}+M_{1}\right)
\end{aligned}
$$


For a fixed pair $\left(f_{0}, f_{1}\right)$ satisfying Equation (A.18) there are only two solutions of System (A.2); both are given by the formula

$$
K^{-1}=\left(\gamma_{0} e_{01}, \gamma_{1} e_{1-1}\right)
$$

and specified by the choice of a branch of the square root:

$$
\gamma_{0}=\sqrt{\frac{f_{1}}{q s\left(r_{1}-1 / r_{1}\right)},} \quad \gamma_{1}=\frac{1}{\gamma_{0} s} .
$$

The column eigenvectors $e_{\nu \epsilon}, \nu=0,1, \epsilon=-1,1$ are defined as follows $\left(M_{\nu 6}-\right.$ $\left.r_{\nu}^{\epsilon}\right) e_{\nu 6}=0$ and $\operatorname{det}\left(e_{\nu 1}, e_{\nu-1}\right)=1$. The complex numbers $p, q, r, s$, satisfying the relation $p s-q r=1$ define transformation between the basis $\left(e_{01}, e_{0-1}\right)$ and $\left(e_{11}, e_{1-1}\right)$ in $\mathbb{C}^{2}$ :

$$
\begin{aligned}
e_{11} & =p e_{01}+q e_{0-1}, \\
e_{1-1} & =r e_{01}+s e_{0-1} .
\end{aligned}
$$

Remark A.5 The numbers $q, s, f_{1} \neq 0$ see the proof below. The comment concerning an ambiguity in Equation A.19) is analogous to that in Remark A.3.

Proof. Consider Transformation (A.21). If $s=0$, then one proves that Equations A.3 and (A.4) are valid, so that we have the unsolvable case of the problem, see Item $3 a$ in Theorem A.1.

If $q=r=0$, then $\left[M_{06}, M_{16}\right]=0$. It is a solvable case of the problem, see Proposition A.2 and Item 2 of Theorem A.1.

If $q=0, r \neq 0$, then Equations a.9 and a.10 are valid: it is a solvable case of the problem, see Proposition A.3 and Item 1 of Theorem A.1.

If $q \neq 0, r=0$, then Equations (A.13) and (A.14) are valid: it is a solvable case of the problem, see Proposition A.4 and Item 1 of Theorem A.1.

If $p=0$, then it is also a solvable case of the problem: see Item $3 b$ of Theorem A.1. This case can be also treated as Item 4 of Theorem A.1 for the parameters $q r=-1$.

Consider now the general case Items $3 b$ and 4 of Theorem A.1 i.e., qrs $\neq 0$. If the problem is solvable, then $K^{-1}$ can be written in the form (A.19) with $\gamma_{0}, \gamma_{1} \in \mathbb{C} \backslash\{0\}$. Since $K^{-1} \in \mathrm{SL}(2, \mathbb{C})$, one using Transformation A.21 proves the second condition in (A.20). Substitution of Equation (A.19) into System (A.2) yields:

$$
\gamma_{1} r\left(r_{0}-1 / r_{0}\right)=f_{0} \gamma_{0}, \quad q \gamma_{0}\left(r_{1}-1 / r_{1}\right)=f_{1} \gamma_{1}
$$

Multiplying Equations (A.21) one obtains

$$
f_{0} f_{1}=q r\left(r_{0}-1 / r_{0}\right)\left(r_{1}-1 / r_{1}\right) .
$$

The second equations (A.20) and (A.22) yield the first equation in (A.20).

To prove that Equation (A.23) is equivalent to Equations A.18 one should notice that the matrix $\left(M_{06}-1 / r_{0}\right)\left(M_{16}-r_{1}\right)$ has the following eigenvectors: $e_{11}$ and $e_{1-1}-\frac{s}{q} e_{11}$ corresponding to the eigenvalues 0 and $q r\left(r_{0}-1 / r_{0}\right)\left(r_{1}-1 / r_{1}\right)$, respectively.

Conversely, by substituting Equation (A.19) into System (A.2) and taking into account Equations (A.20) and (A.21) one finds equations (A.18). 
Remark A.6 Suppose the problem is unsolvable, i.e., Equations (A.3) and (A.4) hold. Define the following matrices:

$$
\tilde{M}_{0 \Delta}=\left(\begin{array}{cc}
1 / r_{0} & \tilde{f}_{0} \\
0 & r_{0}
\end{array}\right), \quad \tilde{M}_{1 \Delta}=\left(\begin{array}{cc}
1 / r_{1} & 0 \\
\tilde{f}_{1} & r_{1}
\end{array}\right)
$$

According to Propositions (A.3) and A.4 System (A.2) for the pair $M_{0 \Delta}, \tilde{M}_{1 \Delta}$ with $\tilde{f}_{1}=0$ and for the pair $\tilde{M}_{0 \Delta}, M_{1 \Delta}$ with $\tilde{f}_{0}=0$.

\section{References}

[1] M. Jimbo, Monodromy problem and the boundary condition for some Painlevé equations, Publ. Res. Inst. Math. Sci. 18 (1982), no. 3, 1137-1161.

[2] B. M. McCoy and S. Tang, Connection formulae for Painlevé functions. Solitons and coherent structures (Santa Barbara, Calif., 1985), Phys. D 18 (1986), no. 1-3, 190-196.

[3] B. M. McCoy and S. Tang, Connection formulae for Painlevé V functions, Phys. D 19 (1986), no. 1, 42-72.

[4] B. M. McCoy and S. Tang, Connection formulae for Painlevé functions. II. The $\delta$ function Bose gas problem, Phys. D 20 (1986), no. 2-3, 187-216.

[5] P. Painlevé, Sur les équations différentielles du second ordre à points critiques fixes, Oeuvres de Paul Painlevé. Tome III. (French) Équations différentielles du second ordre. Mécanique. Quelques documents. Éditions du Centre National de la Recherche Scientifique, Paris, 1975.

[6] K. Okamoto, Isomonodromic deformation and Painlevé equations, and the Garnier system, J. Fac. Sci. Univ. Tokyo Sect. IA Math. 33 (1986), no. 3, 575-618.

[7] A. V. Kitaev, Turning points of linear systems and double asymptotics of the Painlevé transcendents, Painlevé transcendents (Sainte-Adele, PQ, 1990), 81-96, NATO Adv. Sci. Inst. Ser. B Phys., 278, Plenum, New York, 1992.

[8] A. V. Kitaev, Calculation of a nonperturbative parameter in the $\phi_{4}$ matrix model (Russian), Zap. Nauchn. Sem. Leningrad. Otdel. Mat. Inst. Steklov. (LOMI) 187 (1991), 31-39; translation in J. Math. Sci. 73 (1995), no. 4, 430-435.

[9] A. A. Kapaev and A. V. Kitaev, The limit transition $\mathrm{P}_{2} \rightarrow \mathrm{P}_{1}$ (Russian), Zap. Nauchn. Sem. Leningrad. Otdel. Mat. Inst. Steklov. (LOMI) 187 (1991), 75-87; translation in J. Math. Sci. 73 (1995), no. 4, 460-467.

[10] K. Okamoto, Studies on the Painlevé equations. I. Sixth Painlevé equation $P_{\mathrm{VI}}, A n n$. Mat. Pura Appl. (4) 146 (1987), 337-381.

[11] K. Okamoto, Studies on the Painlevé equations. II. Fifth Painlevé equation $P_{\mathrm{V}}$, Japan. J. Math. (N.S.) 13 (1987), no. 1, 47-76.

[12] V. I. Gromak, On the theory of Painlevé's equations (Russian), Differencial'nye Uravnenija 11 (1975), 373-376. 
[13] E. L. Ince, Ordinary Differential Equations, Dover Publications, New York, 1944.

[14] N. Joshi and M. D. Kruskal, A new coalescence of movable singularities in the fourth Painlevé equation, Preprint AM 93/11, University of New South Wales (1993).

[15] A. R. Its and V. Yu. Novokshenov, The isomonodromic deformation method in the theory of Painlevé equations, Lecture Notes in Mathematics 1191, Springer-Verlag, Berlin, 1986.

[16] A. V. Kitaev, Caustics in $1+1$ integrable systems, J. Math. Phys. 35 (1994), no. 6, $2934-2954$.

[17] T. Kawai and Yo. Takei, On the structure of Painlevé transcendents with a large parameter, Proc. Japan Acad. Ser. A Math. Sci. 69 (1993), no. 7, 224-229.

[18] A. R. Its, A. V. Kitaev, and A. S. Fokas, An isomonodromy approach to the theory of two-dimensional quantum gravity (Russian), Uspekhi Mat. Nauk 45 (1990), no. 6(276), 135-136; translation in Russian Math. Surveys 45 (1990), no. 6, 155-157.

[19] A. S. Fokas, A. R. Its, and A. V. Kitaev, Discrete Painlevé equations and their appearance in quantum gravity, Comm. Math. Phys. 142 (1991), no. 2, 313-344.

[20] A. S. Fokas, A. R. Its, and A. V. Kitaev, The isomonodromy approach to matrix models in 2D quantum gravity, Comm. Math. Phys. 147 (1992), no. 2, 395-430.

[21] R. Narayanan and C. A. Tracy, Holonomic quantum field theory of bosons in the Poincare disk and the zero curvature limit, Nuclear Phys. B 340 (1990), no. 2-3, $568-594$.

[22] J. Palmer, M. Beatty, and C. A. Tracy, Tau functions for the Dirac operator on the Poincare disk, Comm. Math. Phys. 165 (1994), no. 1, 97-173.

[23] M. Sato, T. Miwa, and M. Jimbo, Holonomic quantum fields. I, Publ. Res. Inst. Math. Sci. 14 (1978), no. 1, 223-267.

[24] M. Sato, T. Miwa, and M. Jimbo, Holonomic quantum fields. II. The RiemannHilbert problem, Publ. Res. Inst. Math. Sci. 15 (1979), no. 1, 201-278.

[25] M. Sato, T. Miwa, and M. Jimbo, Holonomic quantum fields. III, Publ. Res. Inst. Math. Sci. 15 (1979), no. 2, 577-629.

[26] M. Sato, T. Miwa, and M. Jimbo, Holonomic quantum fields. IV, Publ. Res. Inst. Math. Sci. 15 (1979), no. 3, 871-972.

[27] M. Sato, T. Miwa, and M. Jimbo, Holonomic quantum fields. V, Publ. Res. Inst. Math. Sci. 16 (1980), no. 2, 531-584.

[28] M Jimbo, T. Miwa, Ya. Mori, and M. Sato, Density matrix of an impenetrable Bose gas and the fifth Painleve transcendent, Phys. D 1 (1980), no. 1, 80-158.

[29] M. Jimbo, T. Miwa, and K. Ueno, Monodromy preserving deformation of linear ordinary differential equations with rational coefficients. I. General theory and $\tau$ function, Phys. D 2 (1981), no. 2, 306-352. 
[30] M. Jimbo and T. Miwa, Monodromy preserving deformation of linear ordinary differential equations with rational coefficients. II, Phys. D 2 (1981), no. 3, 407-448.

[31] H. Umemura, On the irreducibility of Painlevé differential equations, Sūgaku 40 (1988), no. 1, 47-61 (Japanese). Translated in Sugaku Expositions 2 (1989), no. 2, $231-252$.

[32] H. Watanabe, Birational canonical transformations and classical solutions of the sixth Painlevé equation, Ann. Scuola Norm. Sup. Pisa Cl. Sci. (4) 27 (1998), no. 3-4, 379-425 (1999).

[33] A. A. Bolibruch, The Riemann-Hilbert problem and Fuchsian differential equations on the Riemann sphere, Proceedings of the International Congress of Mathematicians, Vol. 1, 2 (Zurich, 1994), 1159-1168,Birkhauser, Basel, 1995.

[34] H. Bateman and A. Erdelyi, Higher Transcendental Functions vol 1 and 2 (New York: McGraw-Hill), 1953-1955.

[35] A. V. Kitaev, The justification of asymptotic formulas that can be obtained by the method of isomonodromic deformations (Russian), Zap. Nauchn. Sem. Leningrad. Otdel. Mat. Inst. Steklov. (LOMI) 179 (1989), 101-109; translation in J. Soviet Math. 57 (1991), no. 3, 3131-3135. 